\title{
Reducing energy waste through eco-aware everyday things
}

\author{
Juan López-de-Armentia ${ }^{\mathrm{a}}$, Diego Casado-Mansilla ${ }^{\mathrm{a}, *}$, Sergio López-Pérez ${ }^{\mathrm{b}}$ and \\ Diego López-de-Ipiña ${ }^{\mathrm{a}}$ \\ ${ }^{a}$ Deusto Institute of Technology - Deusto Tech, University of Deusto, Bilbao, Spain \\ ${ }^{\mathrm{b}}$ University of Deusto, Bilbao, Spain
}

\begin{abstract}
Society wastes much more energy than it should. This produces tons of unnecessary $\mathrm{CO}_{2}$ emissions. This is partly due to the inadequate use of electrical devices given the intangible and invisible nature of energy. This misuse of devices and energy unawareness is particularly relevant in public spaces (offices, schools, hospitals and so on), where people use electrical appliances, but they do not directly pay the invoice to energy providers. Embedding intelligence within public, shared appliances, transforming them into Eco-aware things, is valuable to reduce a proportion of the unnecessarily consumed energy. To this end, we present a twofold approach for better energy efficiency in public spaces: 1) informing persuasively to concerned users about the misuse of electronic appliances; 2) Customizing the operating mode of this everyday electrical appliances as a function of their real usage pattern. To back this approach, a capsule-based coffee machine placed in a research laboratory has been augmented. This device is able to continuously collect its usage pattern to offer feedback to coffee consumers about the energy wasting and also, to intelligently adapt its operation to reduce wasted energy. To this aim, several machine learning approaches are compared and evaluated to forecast the next-day device usage.
\end{abstract}

Keywords: Smart everyday objects, social devices, eco-awareness, energy-efficiency, coffee machines, predictive models

\section{Introduction}

In the last decade, attention to energy efficiency in household and office appliances has increased. The current estimations of the residential electricity consumption by these devices is between $10 \%$ and $15 \%$, while the overall energy use of these products is expected to grow [1]. A big concern is that the residential energy demand in some countries has grown steadily in recent years, well above other European countries. Indeed, they attain more than $15 \%$ of its overall energy [2].

The sheer number of devices deployed in our everyday settings (home, city or work) exhibit different modes of operation as well as different duty cycles. These differences correspond to diverse power consumption for each device and for each operating mode performed. Thus, some devices continuously operate in the Active mode (i.e. all the time connected to the mains), e.g. the telephone. Others like television, toothbrush charger or an electric coffee machine operate in the Standby mode consuming steadily some Watts. The standby power refers to the energy consumed by electronic and electrical appliances while they are switched off (but they are designed to draw some power in this mode). Such power is consumed by internal or external power supplies, remote control receivers, pressure pumps,

\footnotetext{
${ }^{*}$ Corresponding author: Diego Casado-Mansilla, Deusto Institute of Technology, University of Deusto, Avda. Universidades 24, 48007, Bilbao, Spain. E-mail: dcasado@ deusto.es.
} 
keep ready-engines, light displays, or energized circuits when the device is plugged-in but switched off. Finally, there are other devices that do not require energy at all, even if they are connected (No-power mode). Examples of these devices are a simple radio, a hair-dryer or an iron.

The reduction of the overall energy consumption due to the Standby mode is one of the most prominent research topics within the sustainability field. A study of Nipkow et al. [3] demonstrated that the standby power used by these everyday appliances can be as high as 10 to 15 Watts per device or more. This amount leads to wasting around the $2 \%-20 \%$ of the total residential consumption and it represents $1 \%-$ $2 \%$ of the world's $\mathrm{CO}_{2}$ emissions [4]. In fact, if such power leakages at home or office appliances were aggregated at the level of a country or even continents, the amount becomes much more significant. These findings gave place in 1999 to the first energy regulations following the One-Watt-Initiative [5]. This idea, created by governments agencies and backed by the International Energy Agency (IEA), restricts the standby power of new equipment to not more than 1 Watt from 2010, and 0.5 Watts from 2013. Thus, industry, academia's researchers, and even governments are promoting the design of more sustainable and greener devices.

To figure out where and what device leakages energy in our settings is not straight-forward. On the one side, the different operating modes, despite well categorized, and their associated consumption are not obvious to deduce $[1,4,6]$. This is because distinct manufacturers apply different designs for each devices and the associated operating modes are not clearly specified in data-sheets. Therefore, it is hard to devise general-purpose technical solutions to reduce energy consumption for specific devices.

On the other side, energy consumers (ordinary people at home or work) remain completely left out of the understanding of device's power consumption. This lack of understanding is mainly due to the intangible nature of electricity, which difficulties the people efficiency-awareness and in consequence, the hoped task of reducing energy consumption with human actions (e.g. unplug the unused devices). Reflecting on this last statement, we think that eco-appliance's designers are wrong if they expect that end-consumers use the appliances in the same efficient way as these were designed.

This work is motivated by these shortcomings. Our approach to tackle them is twofold: 1) We propose that everyday consumer objects should be able to motivate people to take more and better eco-actions by making visible and understandable their energy leakages, thus increasing the energy-awareness. 2) We deem that everyday collective appliances should incorporate some intelligence to operate differently as a function of its real usage pattern (i.e. automatically switching among available operating modes during the day, in order to save the most energy). We deem that these two approaches should be combined since the cooperation of people and smart technology can bring about a more sustainable behavior change both from people and electrical appliances. Therefore, the main article's contribution is to demonstrate that the previous statemens are true. Indeed, we validate the latter by comparing three machine learning methods in order to conclude which of them is the most appropriated to be applied within Eco-aware everyday things.

Efficiency and sustainability related literature focuses its e-conservation approaches in individuals awareness, in their home's automation (e.g. HVAC or temperature), and in the individual reduction of ecological footprint [7,8]. This work borrows some of their findings, but it is focused on those devices or appliances which are deployed in public spaces (e.g. work-offices, malls, cities, etc.) and which are therefore shared by several people. Another assumption is that the aimed devices must feature different operating modes (i.e. Active, Standby and No-power mode) that can be controlled by users. Examples of such devices are coffee machines, printers, public interactive screens, or public lighting, to mention some.

To validate our hypothesis, we have conducted a three month energy-data collection over a capsulebased coffee machine which is placed in our laboratory. This monitoring is performed to analyze how 
laboratory-workers use the device under-study and to devise solutions to reduce unnecessarily wasted energy (i.e. people awareness and technical strategies) when device misusing occurs. ${ }^{1}$ In a first analysis of the collected data, we observed a random use of the coffee-maker, thus no rationale was found in terms of the device usage: 1) different number of coffees per day, 2) these coffees were prepared at different hours during the working-day, 3 ) people applied different usages - operating modes -, when preparing a hot-drink, i.e. some left the coffee-maker on Standby, and others left the device switched-off (No-power mode) after its utilization. Such randomness is translated into a non negligible waste of energy since, ideally, the device operation should be adapted to the expected number of coffees to be prepared. Thus, depending on the hour of the day and the frequency of coffee intakes, the coffee maker must remain in Standby mode - high number of coffees -, or in contrast, to be switched off - low activity.

The structure of the article is as follows. Section 2 reviews the Internet of Things context in which the presented approach is based. There, the presence of smart everyday objects in Social Networks is analyzed. Section 3 reviews the human-behavior strategies to increase energy awareness. Section 4 is devoted to justify the need of embedding more intelligence into everyday shared appliances to save energy. Since a coffee-machine has been augmented as a proof-of-concept, in this section we also explain how such device operates, we address the rationale for real energy-efficient devices, and we formalize the problem statement to avoid leakages. Section 5 describes the methodology for the data-collection process. Section 6 approaches three different machine learning methods to save energy with the coffeemachine. In Section 7 we analyze the results obtained for each of the proposed methods. Section 8 overviews the related work. Finally, Section 9 is dedicated to discuss the results, it summarizes the article and it opens the future research lines to conserve more energy in public spaces.

\section{An environmentally friendly internet connected world}

Communication courses held at university or school are intended to teach people, i.e. human beings, to express in public their feelings or knowledge in a clear and understandable manner. Now with the advent of the computer's era, new computational actors, in the shape of well known everyday objects, come also to express their feelings and they should be adequately prepared to communicate such information in a comprehensible way for humans and other machines. These computational actors are spreading widely. An example of this statement is a Jim Morrish's report [9] who foretell more than 16 billion connectable consumer devices in the next ten years, which means a worldwide average of between 0.8 and 5.8 devices for each person alive in 2020 .

These facts, unthinkable years ago, are occurring thanks to the miniaturization of computers and the decrease of prices without losing any computational power (recall Moore's law [10]). Currently, it is affordable to embed intelligence within these everyday objects and in consequence, make real the Weiser's disappearing computer dream [11].

In this new Internet of Things world, the smart everyday things are able to sense their surrounding information, and to express the acquired knowledge in an interactive, multimodal and comprehensible way in order to make people's everyday activities more bearable. This idea is not naïve at all, since human beings can not perceive all the information around them, either because they are not physiologically able to "sense" such information (e.g. $\mathrm{CO}_{2}$ particles or energy consumption of home appliances), or either because they are not physically in the appropriate place to be aware of such information (imagine

\footnotetext{
${ }^{1}$ For this article,the term 'misuse' is applied when is more efficient to perform an operating mode than another for energy reduction proposes.
} 
somebody who has left the water tap opened in the bath and forgets to close it when moving to another room).

In our opinion, Internet-connected objects may contribute to reduce energy consumption by giving them an 'active-voice' towards energy efficiency. Thus, the remain of the article unveils how intelligent everyday objects and social channels may be combined to fill the gap between electronic appliances and human beings.

\subsection{Eco-aware smart everyday objects}

F. Siegemund presented in his thesis [12] 'Smart Everyday Objects' as arbitrary items from our everyday environment, such as a table, a chair, or an appliance, augmented with information technology embedded in the design. Similarly, Bohn, Mattern et al. [13] argued that objects become smart when microelectronics and sensing components are endowed within them.

In the field of eco-awareness, there are several physical gadgets and devices which are designed to make visible the energy consumption in real-time. For example, Wattson Solar ${ }^{2}$ the Energy Orb, ${ }^{3}$ TED detective $^{4}$ or Onzo. ${ }^{5}$ Nevertheless, they are far from being Internet-connected smart objects. Furthermore, these commercial devices are conceived for people's settings (i.e. individual use) while we advocate for Eco-aware objects for energy awareness in public spaces.

\subsubsection{Social networks for energy reporting and human-object cooperation}

Most of the communication interfaces between smart everyday things and humans are device's embedded screens, lights or sounds. The interaction through these channels only occurs when users are near to the referred device. Lately, more Internet-connected objects are extending the previous channels making them remotely accessible through IP services. However we reckon that Social Networks are currently a more appropriate channel to really cooperate with them. Firstly, due to the social nature of human beings which eases and makes more natural the communication, and secondly because Social Networks are now experimenting a exponential growth (e.g. Twitter has officially become the next big thing in terms of Internet social phenomena gaining worldwide popularity, with over 400 million users per $\left(\mathrm{day}^{6}\right)$, and therefore it is easy to reach thousands of users with just one 'tweet'. Consequently, we have connected our under-study coffee maker to the Twitter sphere (@Social_coffee ${ }^{7}$ ) in order to make it participate as a new, but active, social entity.

Other previous authors have also given a 'voice' to smart objects in Social Networks. J. Bleecker coined the Blogjects neologism that is meant to focus attention on the participation of objects in the sphere of networked social discourse. Vázquez et al. [14] introduced the concept of Social Devices. More recently, Social Network-connected gadgets have emerged (e.g. Rymble, ${ }^{8}$ Botanicalls ${ }^{9}$ or the Tweetjects deployed in the house that tweets ${ }^{10}$ ). Therefore, we deem that Twitter is a convenient platform to bridge the physical and virtual world, enabling our @ Social_coffee to 'inform' coffee consumers about energy consumption issues, and then promoting eco-awareness.

\footnotetext{
${ }^{2}$ http://www.diykyoto.com/.

${ }^{3}$ http://www.orbenergy.com.

${ }^{4} \mathrm{http}: / / \mathrm{www} \cdot$ theenergydetective.com/.

${ }^{5}$ http://onzo.com/.

${ }^{6} \mathrm{http} / / / \mathrm{cnet} . \mathrm{co} / \mathrm{KHlg} 8 \mathrm{q}$.

${ }^{7}$ http://twitter.com/\#!/Social_Coffee.

${ }^{8}$ http://www.rymble.com/.

${ }^{9}$ http://www.botanicalls.com/.

${ }^{10} \mathrm{http}: / /$ stanford-clark.com/andy_house.html.
} 


\section{Increasing awareness in public spaces for behavior change}

Energy-leakage awareness in public spaces implies a better education on sustainability and a overall conscience of natural-resources scarceness. Although education is key in this regard, it is very important to put in place technical strategies to make the energy visible and tangible to prevent misusing [8]. However, most existing motivational and conservation approaches are only focused in final-users and individuals' homes, obviating the importance to such eco-awareness in shared places like cities or public buildings.

This problem is due to the general misunderstanding that energy wasting is only an issue of economical ground rather than an overall planet necessity. Next we review some of the approaches that have been devised, mainly by interested governments agencies, to lower the barrier among people and energy awareness. We remark their merits and drawbacks.

The energy suppliers send to the customer's mail-boxes a monthly report of their consumption and fortunately, since few years ago, also the approximate breakdown of the energy consumed by household appliance. However people often forget their compromise to reduce energy if they only receive one feedback per month. Even with the advent of Smart Meters [15], that will entail real-time accessibility to consumption, the reduction will not always occur, since knowing the energy consumed is not sufficient to get a rough idea of actions that could be performed to reduce the bill [16].

Another approach is a guide of good practices for energy saving purposes that is issued each year for the governmental departments of energy. The main intention behind those brochure is to motivate and conscience citizens to change their everyday practices towards more sustainable ones. Although it is a good idea, it suffers from a lack of acceptance. This might be due to an inefficient advertising campaign or to its inappropriate format - in the case of the Spanish Government, it account with almost 100 pages $^{11}$ - for the burden of information that we experienced in our everyday life. In some EU countries, local services also conduct information and motivation campaigns to raise consumers' awareness and encourage them to purchase equipment with low consumption.

A strategy to better understand the appliance's modes of operation and its energy leakages from the consumer side, is to install electricity feedback systems. Froehlich et al. described eco-feedback systems in detail and discussed their effectiveness [17] in households. Unfortunately, there are no hints in the article whether these systems could be also useful in public spaces. In the opposite side, electricity suppliers and researches on the field - in an attempt to increase the percentage of Eco-aware people, are approaching different strategies that could be applied when the awaited Smart Meter has arrived. For instance Mankoff et al. [18] leaned towards specific energy-topic social networks. In this kind of networks people within similar settings (e.g. people in the same flat with a similar number of inhabitants) would interchange their consumption in order to participate in fair competitions to promote a behavior change to save energy. Similarly in [19] the authors have conducted an empirical experiment in a neighborhood to demonstrate that more reduction is possible if social norm and nudge, i.e. telling the people what others do, is applied.

With these premises, we conclude that is difficult to be familiarized with energy. Indeed, it is not an easy task to convince the end-users about the economic and environmental benefits of adopting energy efficiency practices, particularly when the quantum of saving is not high at the individual level and it would occur in places where the individual does not pay invoices.

\footnotetext{
${ }^{11}$ http://www.idae.es/index.php/id.542/relcategoria.1161/relmenu.64/lang.uk/mod.pags/mem.detalle.
} 


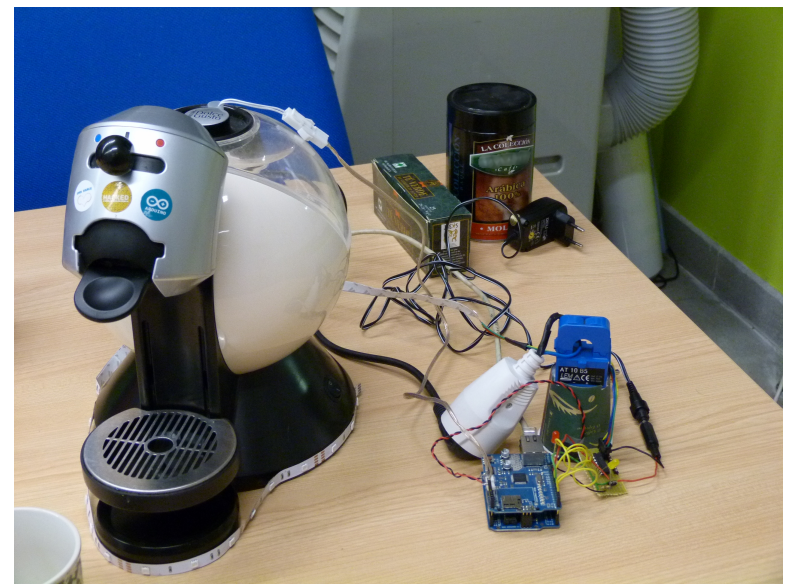

Fig.1. @Social_Coffee.

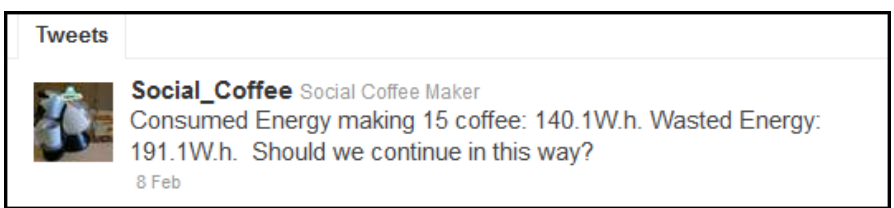

Fig. 2. A @Social_Coffee 'tweet' summarizing the daily energy consumed during a work-day.

The approach that we have devised to make our laboratory-colleagues aware about the energy leakages and device misusing has been to augment a coffee machine with electronics and an Internet connection that allows it to have a 'green-voice' in Social Networks. The idea was to convert such a dump electrical device into a social and affective entity to be able to interact with the crowd and not only individuals near to the coffee-maker. The first ideas issued about the more appropriate channels to make objects communicate, were those that most people could perceive and be aware of in real time. In the case of the @Social_coffee (the alter-ego of implemented proof-of-concept to validate our hypothesis), it was decided to cover such communication need with Twitter. By using this platform, smart everyday objects and human beings will be placed at the same communication layer, and can mutually learn and cooperate with each other. Figure 1 shows a picture of the augmented device and its electronics.

The @Social_Coffee amounts more than 50 followers (covering roughly the 100\% of our laboratoryresearchers). Thus, whenever the Eco-aware social object posts its everyday energy-state, ${ }^{12}$ as Fig. 2 shows. Such information is rapidly spread among its followers to motivate them to perform a more efficient use of the shared appliance.

\section{Coffee machines: Electrical devices with eco-awareness needs}

The stock of coffee machines in EU is estimated to be 100 million units, consuming 17.000 million $\mathrm{kWh}$ per year and causing electricity cost of about 2.500 Millions of Euro. The estimated sales are about

\footnotetext{
${ }^{12}$ The @Social_Coffee 'tweets' the comparison between the effective energy consumed vs. the energy wasted. Besides it offers related information like remaining water level, the coffee cups filled in a working-day or even eco-tips to prevent energy leakages.
} 


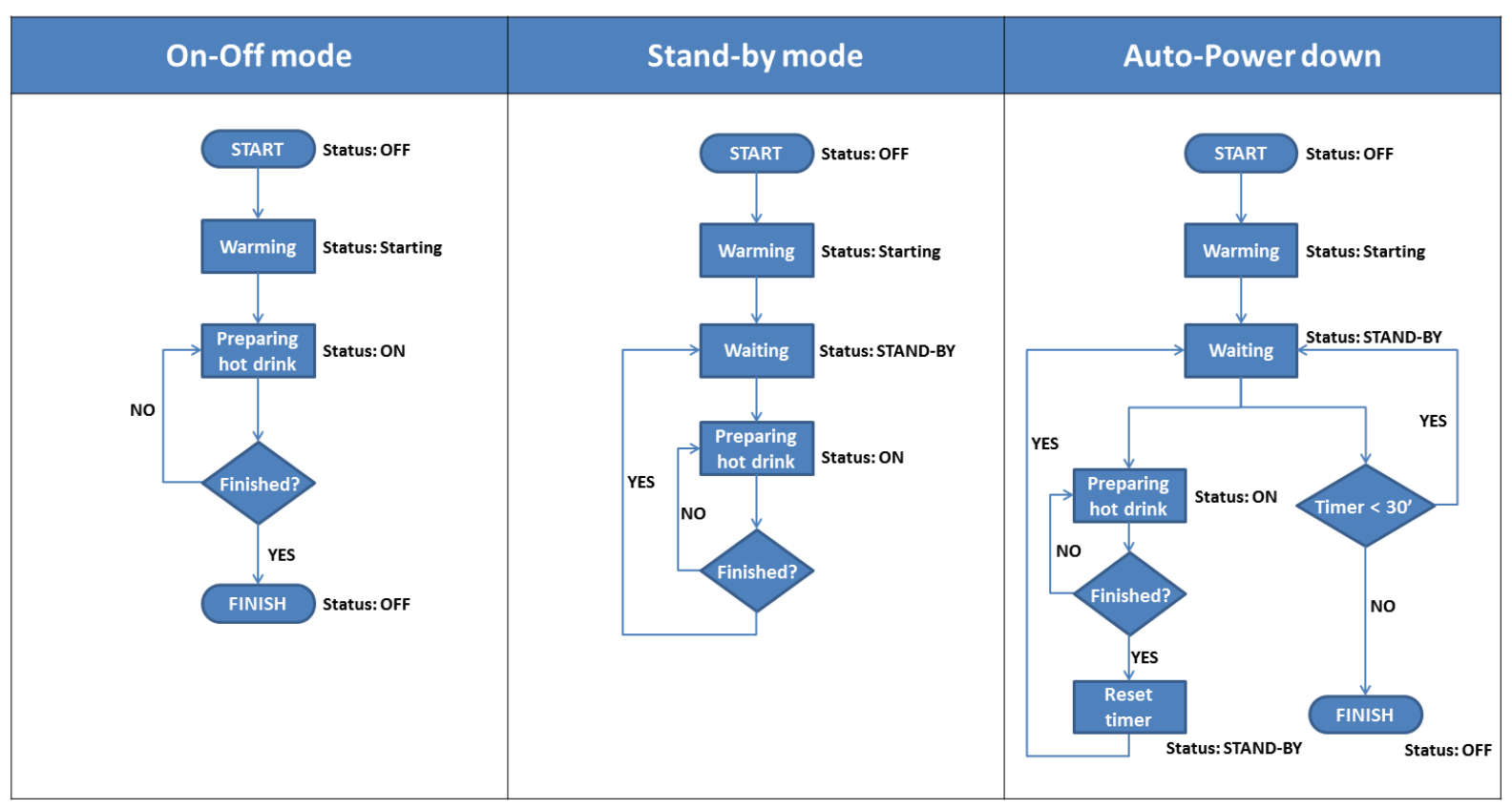

Fig. 3. Coffee-maker operating modes.

20 million coffee machines by year, thereof 10 million traditional coffee machines and 8 million espresso and filter-pad machines [20]. In [3], it is stated that coffee machines use large amount of electricity for permanently keeping hot and Standby modes. Surprisingly, some espresso machines usually have higher consumption than A-class ovens or A++ fridges. The same authors argue that by applying simple measures as auto-power-down, better insulation of boilers and low standby consumption, the energy efficiency can be strongly enhanced. Although in a similar report from SAFE group [21] it is stated that the capsules coffee machine's consumption is relatively low, $40 \mathrm{kWh}$ per year, we think it is possible to reduce this consumption further by applying more intelligence over these devices, beyond static ecodesign, according to their usage pattern.

\subsection{Operating modes - The case of coffee machines}

The under-study coffee-maker is categorized within the group of devices and appliances which operate in different modes and, since located in public spaces, are shared by several people.

This coffee machine, in particular, is designed to operate in two different modes: The On-Off mode (a combination of previously surveyed Active and No-power modes) and the Standby mode, also referred to as 'keeping hot' [22]. However, boosted by the EU recommendation [21], this model of coffee machine is also programmed with an on-the-self energy efficient mode, which is called Auto-power down mode.

Figure 3 shows three state diagrams of the modes of operation that the off-the-shelf capsule-based coffee machine performs. Before explaining them, let's first recall that this coffee-maker model has a start button in order to switch it on and switch it off, and a LED light that shifts from blinking to steady to warn users when the device is ready to be used.

The process of operating in On-Off mode is graphically described in the left side of Fig. 3. A user has to press the start button since the device was switched off. After few seconds of engine warming (blinking 


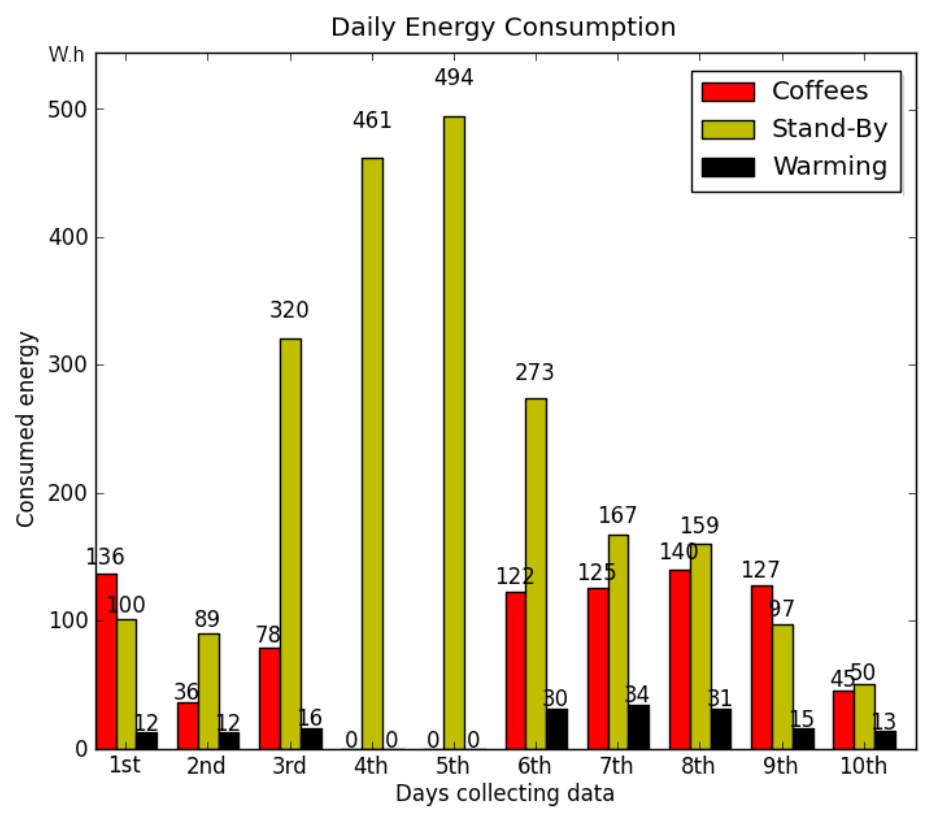

Fig. 4. Energy consumed during 10 consecutive days.

LED light), the coffee-machine is ready to use. The user prepares a hot drink and then switches off the device again. This mode does not consume energy whilst idle periods, but the coffee-maker water pressure system consumes a significant amount of energy when it is again switched on for the next drink.

The second operating mode is the Standby mode (the middle of Fig. 3). A user will always find the coffee-maker ready-to-use because the appliance is permanently working. Thus, no warming time is needed, as before, due to the periodical energy peaks that the device consumes to maintain the engine's pressure.

When the last operating mode, Auto-power down mode, it is activated, the coffee machine is able to itself completely switch off if it detects an inactivity period of more than 30 minutes.

As it was explained in [23], these modes are randomly detected with no rationale during a work-day in our laboratory due to the variety of hot beverages, consumer preferences and time to prepare a drink. The non-existent information about how use the coffee machine energy efficiently causes users indifference (i.e. the people gives not importance to the choice of a convenient operating mode to save energy). As an example, observing the Fig. 4, one might be confused when realised that the days the fewer coffees have been made (zero coffees the 4th and the 5th), the most energy was wasted. This fact was due to someone absentmindedness (i.e. forgetting the appliances on when these are not going to be used at all such as week-ends or after-work periods).

Indeed, we can assert that this confusing behavior is worldwide generalized. Figure 5 shows a completely different coffee machines switching behavior at work on surveyed countries. ${ }^{13}$ That can be an evidence of absence of rules to efficiently use the coffee machines.

Therefore, these findings led us into the definition of a new mode, called Eco-aware mode, as an intelligent combination of On-Off mode and Standby mode. This means that the coffee-maker would

\footnotetext{
${ }^{13} \mathrm{http} / / /$ www.eceee.org/Eco_design/products/Lot25_non_tertiary_coffee_machines.
} 


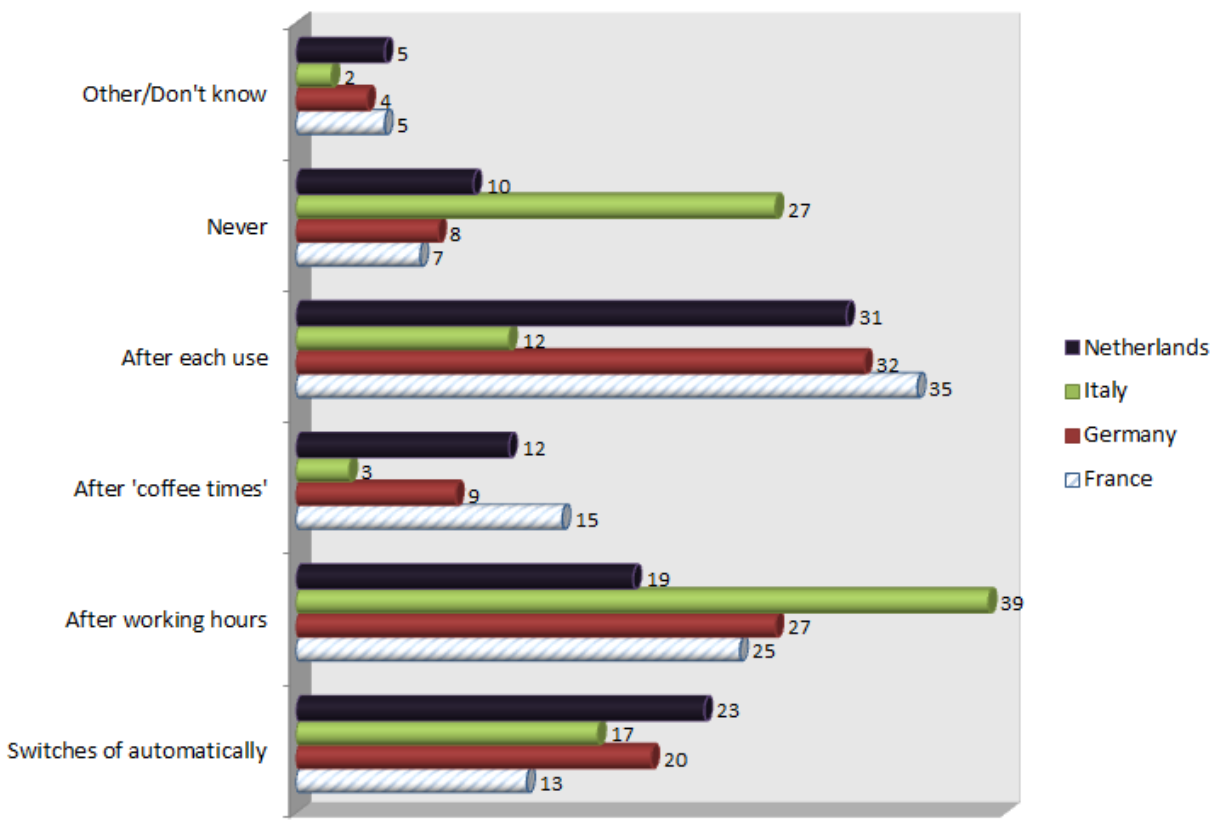

Fig. 5. Coffee machines switching behaviour at work in France, Germany, Italy and Netherlands.

have the capacity to automatically change from one operating mode to another in an efficient way. The next section is devoted to formalize the rationale behind the choice of an appropriate mode in each period, and the parameters which influence such decision.

\subsection{Switching between operating modes - The problem statement}

More than thirty people work in the laboratory where the coffee-maker is deployed. This led to different people schedules, an therefore a work activity that ranges from 7 a.m. to 7 p.m. We denote this range by $D$. To demonstrate the assumption that a Eco-aware mode is better than before presented modes, we advocate to divide $D$ in time slots of $T_{s l}$ seconds each. The goal is to determine the minimum number of coffees, $n$, that must be prepared per slot to intelligently commute from the On-Off mode ont the Standby mode in order to save energy.

To such extent we equate Eq. (1) the energy consumed in a whole working-day, $D$, if only the On-Off mode had been applied $\left(E_{n f \mid T_{s l}}\right)$ and if solely the Standby mode had been applied $\left(E_{s b \mid T_{s l}}\right)$.

$$
E_{n f \mid T_{s l}}=E_{s b \mid T_{s l}}
$$

For the sake of easing the formulas understanding, we do not explain in deep the meaning of all temporal parameters. Let consider them as scalar times, measured in seconds, that have been averaged after the data-collection phase (see next Section). These parameters are extracted from the process of preparing coffee in the under-study coffee-maker model: $T_{c f}$ is the average time to prepare a coffee; $T_{s b}$ is the time between energy keeping hot peaks in Standby mode; the time to boot up the coffee-maker engine is denoted by $T_{s t} ; T_{\text {peak }}$ is the time that energy keeping hot peaks lasted; and $T_{a c f}$ refers to the average time that the coffee-maker needs to be ready after prepare a coffee. 
The energy consumed in the On-Off mode does not care about $T_{s l}$. It only depends on $n$ and can be calculated with Eq. (2):

$$
E_{n f}=n\left(\overline{T_{s t}}+\overline{T_{c f}}\right)
$$

In contrast, to calculate the energy consumed by the Standby (Eq. (4)) we have to first mathematically define $n_{\text {peak }}$ (Eq. (3)). In this case, this is mandatory since the number of energy peaks triggered by the Standby mode depends on $T_{s l}$.

$$
n_{\text {peak }}=\frac{T_{s l}-n\left(\overline{T_{a c f}}+\overline{T_{c f}}\right)}{\overline{T_{s b}}}+1
$$

Therefore, we are now ready to define the energy consumed by the Standby mode as:

$$
E_{s b \mid T_{s l}}=n\left(\overline{T_{c f}}\right)+\left(n_{\text {peak }} * \overline{T_{\text {peak }}}\right)
$$

With these formulas is easy to work out $n$ to know the minimum number of coffees that should be prepared to shift from the On-Off to the Standby mode. We have advocated to divide the working day into slots of one hour $\left(T_{s l}=3600\right.$ seconds), then $n=2.3$ coffees.

\subsubsection{The Baseline and the Optimal mode}

With the previous information, the objective for the Eco-aware mode is "to automatically derive a predictive model which would infer when $n \geqslant 2.3$ for each of the slots during $D$ to minimize the energy consumed". In order to test the performance of the selected predictive models, in the article we evaluate them against the empirical usage-data that we have collected. This real data is denoted as the baseline. Therefore, whatever minimal energy reduction approach for the coffee maker would outperform it.

In the opposite side, we have manually devised an Optimal mode for the coffee-machine usage dataset. That is, a model that always predict appropriately when the coffee machine would be in a mode or another to save the most of the energy. As was demonstrated before, if we divided the working day in slots of one hour, this Optimal mode will always forecast when the number of coffees exceed the threshold ( $n=2.3$ coffees) for each of the slots. This simulated mode is the objective that the proposed predictive models should attain. Then, it is used together with the baseline to evaluate each of them.

\section{Data collection}

We conducted a process through which we collected coffee-maker usage data during 81 days (i.e. between the 2nd of February and the 24th of April, 2012).

\subsection{Apparatus design}

The instrumentation used for this data collection process is detailed next:

1. The everyday object that has been converted into a smart device, is an off-the-shelf capsule-based coffee machine, namely Dolce Gusto ${ }^{\circledR}$. 
2. To tackle the coffee-maker consumption, a LEM_AT_10_B5 current sensor has been used. This sensor is self-powered by induction, its maximum amperage is $10 \mathrm{~A}$, and the output, that varies from 0 to 5 volts depending on the current that is flowing through it, is then processed by an Arduino microcontroller. Assuming a mains voltage of $220 \mathrm{~V}$, a power factor (PF) as the unit, and setting the consumption time as $t$, the consumed energy can be worked out as:

$$
\begin{aligned}
& P=V * I * P F \\
& E=P * t
\end{aligned}
$$

3. An Ethernet shield attached to the Arduino allows Internet connection and to store in a micro-SD the device usage data. This data is daily separated in files (each file name corresponds to the date when the data was collected). In each of them we store the timestamped coffee-maker's activity and its associated energy consumption as explained below.

The coffee-maker activity is described within the files in separated lines. Whenever a energy consumption event is detected a new line is written with the format that is explained hereafter:

$$
\text { Start time }_{\text {tim }} \mid y:\left[\text { Power }_{K w h} ; ;\right] x:\left[\text { Peaks }_{\text {timestamps }} ; ;\right] \mid \text { Consumption }_{\text {type }} \mid \text { Duration }_{\text {secs }}
$$

and a sample of it:

$$
30685|\mathrm{y}:[1037.4 ; 1256.5 ; 1247.9 ;] \mathrm{x}:[30685 ; 30686 ; 30687 ;]| \text { PEAK|3.89| }
$$

Here the Start $_{\text {time }}$ refers to the exact time in seconds, counting from 12 a.m., where a consumption event is detected (e.g. 30685 in the sample refers to a PEAK drawn between 8 a.m. and 9 a.m.); The $y$ vector shows the power consumption measured in $\mathrm{kWh}$ during the detected event. The longer the time that the coffee-maker is operating, the larger the vector size; The $x$ vector values correspond to the timestamps, also measured in seconds from 12 a.m., where each of the previous power measures have been derived; The Consumption type $_{\text {e }}$ differentiates the sample among the three types of consumptions and is pivotal to detect the operating mode of the coffee maker: START_TIME identifies the consumption event of warming the coffee-maker after switching it on; $\mathrm{COFFEE} \mathrm{determines} \mathrm{that} \mathrm{a} \mathrm{cup} \mathrm{is} \mathrm{being} \mathrm{prepared;} \mathrm{while}$ PEAK] is drawn whenever a keeping hot consumption event occurs. Finally, the Duration $_{\text {secs }}$ refers to the time in seconds that the sample has lasted.

\subsection{Dataset analysis}

After curating and filtering the raw dataset, ${ }^{14}$ we have kept 41 working days.

Making use of this dataset, we are then able to compute for each of the slot times, days or even weeks: 1) the total energy consumption, 2) when such consumption events are derived and 3) the number of coffees prepared. Going even deeply, it is possible to make a breakdown of consumptions type (PEAK | COFFEE |STAR_TIME) and to determine the previous computations for each of them.

Figure 6 shows two different views of the coffee consumption for the 41 days that the coffee-maker was monitored. On the left graph, the dots denote the exact time when a coffee was prepared, while the right one presents its histogram. In a glimpse, we can see a high concentration of usage in two separated periods, mornings and afternoons. This led us to think that one of the previously presented modes might be more appropriate at certain times than another (i.e. when the number of coffees prepared exceeds the threshold, $n=2.3$ ), thus an intelligent combination should save energy. Nevertheless, the data collected is very irregular if we analyze the dataset as a whole (i.e. treating everyday equally without making assumptions on specific weekly days). This fact can easily lend to inconsistencies or erratic inferences when apply learning and predictive methods over it.

\footnotetext{
${ }^{14}$ We have discarded 12 weekends, Easter holidays and other outliers or biased samples.
} 

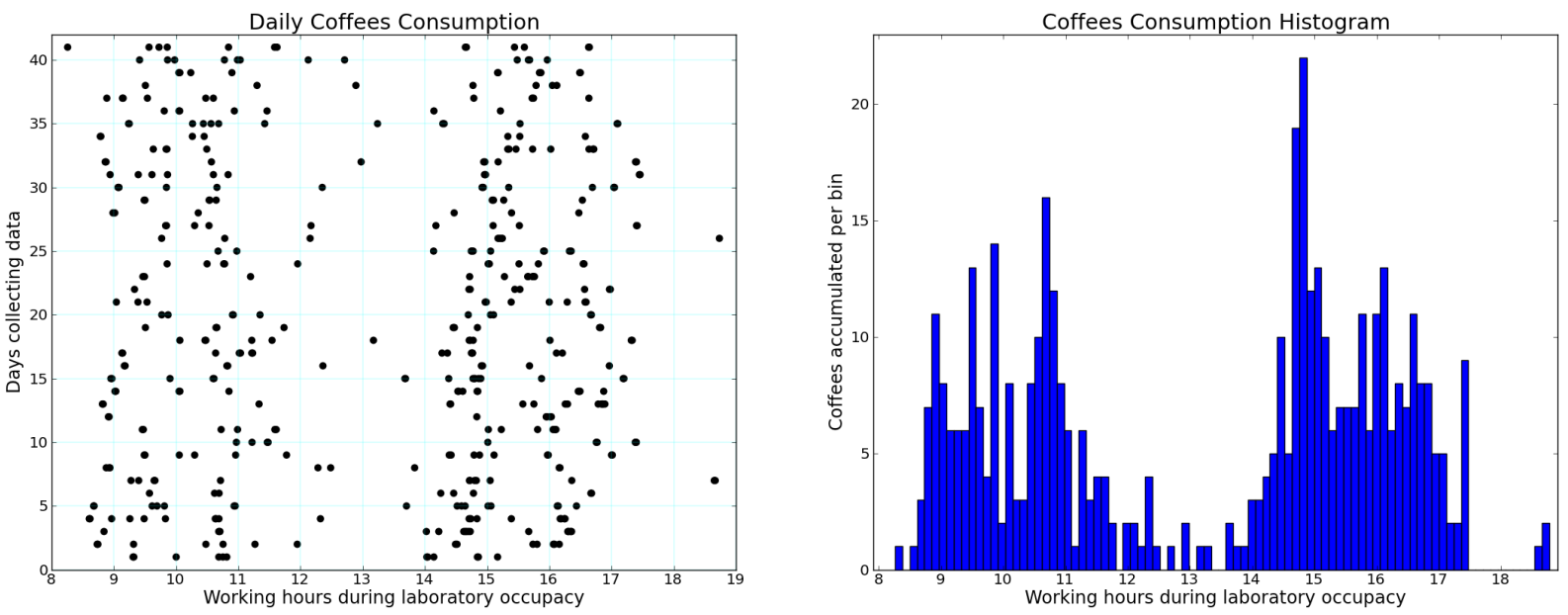

Fig. 6. Coffees distribution during 41 work-days and its associated histogram to observe the number of coffees prepared by slot-time $\left(T_{s l}=3600 \mathrm{~s}\right)$.

\section{Machine learning methods to predict operating mode}

In this section we review three inference approaches, an heuristic approach, Naïve Bayes and Time Series - ARIMA, that have been simulated over the coffee machine's usage data. The idea behind is to compare the performance exhibited of each of the learning approaches when they forecast an appropriate operating mode to be applied for each of the slots. To such extend, we use the total energy consumption and a well known accuracy and quality metrics: the Mean Absolute Error (MAE), Root Mean Square Error (RMSE) and Mean Absolute Square Error (MASE).

As has been explained before, we use a 41 day dataset. The first 30 days compose the training set and the remain 11 days form the test set to evaluate the training performed. The 30 days that make up the training set are temporally consecutive and the same is applied with the test set. In the Naïve Bayes method and the heuristic approach, we would not have taken in account the temporality, because it does not affect the model. However, as in time series the temporality is essential, we have then respected the order of the days to obtain results on the basis of the same conditions.

We have performed the training and testing processes in two ways for each of the training models. The first one, considers the whole day (from 7 a.m. to 7 p.m., omitting the hours when the laboratory is closed). The second one, considers the day separated in morning and afternoon (the former from 7 a.m. to 1 p.m. and the latter from 1 p.m. to 7 p.m.). We have evaluated this two scenarios since the number of coffees prepared and, therefore, their probability distribution are different. Following, for each of the machine learning methods, we present the scenario that yields better performance results.

\subsection{Heuristic approach}

This probabilistic approach has been devised reasoning about how the probability of maintaining the coffee-maker on, $p\left(m_{o n}\right)$, varies according to different parameters. The equation is as follows:

$$
p\left(m_{o n} \mid c_{a c c}, \overline{c_{a v}}, s_{r}, s_{T}\right)=p\left(m_{s b}\right) \times \frac{1-\left(c_{a c c} / \overline{c_{a v}}\right)}{s_{r} / s_{T}}
$$

Equation (7) determines the $p\left(m_{o n}\right)$ given the occurrences of $c_{a c c}$ (the number of coffees prepared in a day until a certain slot; $\overline{c_{a v}}$ (the mean of coffees prepared per day); $s_{r}$ (the number of remaining periods 


\begin{tabular}{|c|c|c|c|c|c|c|c|c|c|c|c|c|}
\hline Days & $7 \mathrm{~h}$ & $8 h$ & 9h & $10 \mathrm{~h}$ & $11 \mathrm{~h}$ & $12 \mathrm{~h}$ & $13 \mathrm{~h}$ & $14 \mathrm{~h}$ & $15 \mathrm{~h}$ & $16 \mathrm{~h}$ & $17 \mathrm{~h}$ & $18 \mathrm{~h}$ \\
\hline 1 & 0,00 & 0,06 & 0,21 & 0,28 & 0,05 & 0,00 & 0,00 & {$[0,38$} & 0,26 & 0,34 & 0,05 & 0,00 \\
\hline 2 & 0,00 & 0,06 & {$[0,37]$} & 0,17 & 0,02 & 0,00 & 0,00 & {$[0,38]$} & 0,26 & 0,27 & 0,04 & 0,00 \\
\hline 3 & 0,00 & 0,06 & 0,21 & 0,28 & 0,02 & 0,00 & 0,00 & {$[0,38]$} & 0,37 & 0,20 & 0,00 & 0,00 \\
\hline 4 & 0,00 & 0,06 & {$[0,37]$} & 0,28 & 0,00 & 0,00 & 0,00 & {$[0,38]$} & {$[0,37]$} & 0,34 & 0,04 & 0,00 \\
\hline 5 & 0,00 & 0,06 & {$[0,37]$} & 0,38 & 0,02 & 0,00 & 0,00 & 0,32 & 0,20 & 0,20 & 0,03 & 0,00 \\
\hline 6 & 0,00 & 0,06 & 0,29 & 0,06 & 0,00 & 0,00 & 0,00 & {$[0,38$} & 0,31 & 0,34 & 0,05 & 0,00 \\
\hline 7 & 0,00 & 0,06 & 0,37 & {$[0,38]$} & 0,11 & 0,00 & 0,00 & {$[0,38$} & 0,31 & 0,27 & 0,03 & 0,00 \\
\hline 8 & 0,00 & 0,06 & {$[0,37]$} & {$[0,49]$} & 0,02 & 0,00 & 0,00 & {$[0,38]$} & 0,31 & 0,34 & 0,03 & 0,00 \\
\hline 9 & 0,00 & 0,06 & {$[0,37]$} & 0,17 & 0,00 & 0,00 & 0,00 & {$[0,38]$} & 0,37 & 0,27 & 0,02 & 0,00 \\
\hline 10 & 0,00 & 0,06 & 0,29 & 0,06 & 0,00 & 0,00 & 0,00 & {$[0,38]$} & 0,37 & 0,20 & 0,03 & 0,00 \\
\hline 11 & 0,00 & 0,06 & 0,21 & 0,28 & 0,05 & 0,00 & 0,00 & {$[0,38]$} & 0,26 & 0,20 & 0,01 & 0,00 \\
\hline
\end{tabular}

Fig. 7. Comparison of heuristic approach results and Optimal mode operation

from the current slot until the end of the working-day $(7$ p.m. in the whole day case, while 1 p.m. or 7 p.m. whether the half day was applied); $s_{T}$ (refers to the 12 hours when the laboratory is inhabited, from 7 a.m. to 7 p.m., and in the case of dividing the day in morning and afternoon, from 7 a.m. to 1 p.m. or from 1 p.m. to 7 p.m.). $p\left(m_{s b}\right)$, is the probability that the coffee-maker prepares more than 2.3 coffees (the limit of coffees to switch from one operating mode to another) in a certain period.

$p\left(m_{o n}\right)$ should decrease whenever the accumulated of coffees prepared approaches to $\overline{c_{a v}}$. By contrast, this probability should increase whenever the remaining slots $\left(s_{r}\right)$ is close to reach the end of $s_{T}$.

Considering that the prepared-coffees distribution, either in the morning and in the afternoon, corresponds with a normal distribution $\mathcal{N}(0, \sigma)$, we can perform a hypothesis test to infer when to switch from a operating mode to another. To this aim, we have defined a threshold value, $\alpha$, which corresponds with the confident bound to retain or reject the switching-hypothesis. Therefore, a value of probability in Eq. (7) lower than $\alpha$ means that the null hypothesis, $H_{0}$, would be true ( $H_{0}$ defines that the coffee machine switches-off after preparing coffee). While a value of probability greater than $\alpha$ means that $H_{0}$ would be rejected. The alternative hypothesis, $H_{1}$, denotes that the coffee machine is maintained on after preparing coffee.

Figure 7 shows the values acquired by probability in each slot. We can observe three colours: 1) yellow (- - - -) points the false negatives (i.e. the coffee machine would switched off, but it should be maintained on according to the Optimal mode); 2) cyan $(----)$ denotes the false positives (i.e. according to the heuristic approach, the coffee machine would incorrectly maintained on); 3) green (...) means that the prediction of to be maintained the coffee machine on is correct.

The bounds to switch from one operating mode to another have been established by means of the previous hypothesis test with $\alpha=0,37$. This threshold value was determined based on empirical coffeemachine's data-observation.

We observe that there are 5 slots correctly predicted to maintain the coffee-maker on. However, it would be on in 18 periods when it should be operating in On-Off mode.

\subsection{Naive bayes classifier}

A Naïve Bayes Classifier [24] is the most simple Bayesian net model oriented to classification. It assumes all the attributes are independent from each other, so all of them independently contribute to the probability's value. 
Although being a simple algorithm, it has performed quite well in many complex situations. In 2004, an analysis of the Bayesian classification problem revealed that there are theoretical reasons for the apparently unreasonable efficacy of Naïve Bayes classifiers [25]. Furthermore, a later research comparing some classification methods, showed that Bayes classification is outperformed by more current approaches [26].

There are two main conditions to use this method: 1) Big training dataset; 2) Independent attributes.

As mentioned before, we have collected 41 days of usage data, of which we take into account 12 hours per day (from 7 a.m. to 7 p.m.). 30 days were taken for training and 11 days for testing, therefore, the dataset contains $30^{*} 12=360$ lines, that we consider it is an adequate amount of data for training the model.

The model features are the following:

- The time when the period of one hour starts.

- Probability that a coffee-maker prepares more than 2.3 coffees in a certain period of one hour.

- Number of coffees prepared in a day until a certain time.

- Number of coffees prepared per day.

The second condition to use Naïve Bayes algorithm is also applied, because all these features are independent.

The Naïve Bayes method is oriented to classification, so we have defined two classes to represent the prediction of the coffee machine's behavior:

1. No: Switch off the coffee-maker after preparing a coffee.

2. Yes: Maintain the coffee-maker in Standby mode after preparing a coffee.

Weka, ${ }^{15}$ a Java-based machine learning framework has been used to test the classification accuracy of Naïve Bayes algorithm was.

Applying Naïve Bayes we obtain misleading results. The percentage of correctly classified instances is good, $87.88 \%$. There are 116 instances correctly classified as the first class and 0 instances correctly classified as the second class. On the other hand, there are 12 false negatives (i.e. the coffee machine would switched off, but it should be maintained on according to the Optimal mode) and 4 false positives (i.e. according to the heuristic approach, the coffee machine would incorrectly maintained on). Looking at Fig. 8, it can be seen that in all the periods when the coffee machine should be maintained on (pointed as 'Yes'), applying Naïve Bayes method would switched it off.

On Section 7 these results are analyzed in energy terms to determine if this method involves an energy saving.

\subsection{Time series - ARIMA model}

A time series is a set of observations generated time-sequentially $\left(y_{1}, y_{2}, \ldots, y_{t}\right)$ and recorder at a specific time $t$ (period - hours, days, months or years). The basic idea behind time series is that it assumes that past patterns will similarly occur in the future, and therefore are predictable [27]. The patterns correspond with the motion of a variable in a time sequence. In the case we are studying, the time-series corresponds with the fluctuations of hot drinks prepared in a laboratory in each of the time slots in which we divided a work day. The series is then as long as the sample we have collected, 41 working days.

\footnotetext{
${ }^{15} \mathrm{http}: / /$ www.cs.waikato.ac.nz/ml/weka/.
} 


\begin{tabular}{|c|c|c|c|c|c|c|c|c|c|c|c|c|}
\hline Days & $7 \mathrm{~h}$ & $8 \mathrm{~h}$ & $9 \mathrm{~h}$ & $10 \mathrm{~h}$ & $11 \mathrm{~h}$ & $12 \mathrm{~h}$ & $13 \mathrm{~h}$ & $14 \mathrm{~h}$ & $15 \mathrm{~h}$ & $16 \mathrm{~h}$ & $17 \mathrm{~h}$ & $18 \mathrm{~h}$ \\
\hline 1 & No & No & Yes & No & No & No & No & No & No & No & No & No \\
\hline 2 & No & No & No & No & No & No & No & No & No & No & No & No \\
\hline 3 & No & No & Yes & No & No & No & No & № & Yes & Yes & No & No \\
\hline 4 & No & No & No & No & No & No & No & No & No & No & No & No \\
\hline 5 & No & No & No & Yes & No & No & No & № & No & No & No & No \\
\hline 6 & No & No & No & Yes & No & No & No & No & No & No & No & No \\
\hline 7 & No & No & Yes & No & No & No & No & No & No & No & No & No \\
\hline 8 & No & No & No & No & No & No & No & No & No & No & No & No \\
\hline 9 & No & No & No & Yes & No & No & No & No & Yes & No & No & No \\
\hline 10 & No & No & Yes & No & No & No & No & ] & Yes & No & No & No \\
\hline 11 & No & No & Yes & No & No & No & No & №으. & No & No & No & No \\
\hline
\end{tabular}

Fig. 8. Incorrectly classified instances categorized by type of error

There are some time-series forecasting models that can be applied (e.g. Holt-Winters, ARMA, ARIMAX, etc.). They depend on several parameters like the purpose of the estimation, the amount of data, or the type and elements of the series, to cite some [27]. Attending to them, we have selected ARIMA models. ARIMA are, in theory, the most general class of models for forecasting and predict if a time series which can be stationarized by transformations such as differencing and logging. These transformations are pivotal since one of the necessary conditions for applying Box-Jenkins method ARMA [28] - the underlying model of ARIMA -, is the stationary of the time-series, which in practice, is very rarely met.

A nonseasonal "Auto-Regressive Integrated Moving Average (ARIMA)" model is defined as "ARIMA $(p, d, q)$ )" (see Eq. (8)), where:

- $p$ ("Auto-Regressive (AR)" terms) are the number of lags of the differenced series appearing in the forecasting.

- $q$ ("Moving Average (MA)" terms) are the number of lagged forecast errors in the prediction equation.

$-d$ is the number of nonseasonal differences. ${ }^{16}$

As Eq. (8) shows, the ARIMA model is a linear combination of polynomials with $p$ and $q$ degree respectively. Its principle is that each time series performs as a function of past values and may only be explained by means of them.

$$
\phi_{p}(L)(1-L)^{d} y_{t}=\theta_{q}(L) \varepsilon_{t}
$$

Here $y_{t}$ is the series; $\varepsilon_{t}$ is the error; $L$ is the lag operator; $\phi_{p}$ is the AR polynomial of order $p ; \theta_{q}$ is the MA polynomial of order $q$; while $(1-L)^{d}$ denotes the number of nonseasonal differences to make the series stationary.

\subsubsection{Methodology for the Coffee-Maker}

Next, the ARIMA model selection and the statistical methodology selected are presented (according to Box-Jenkins methodology [28]).

\footnotetext{
${ }^{16}$ Time series which needs to be differenced to be made stationary is said to be an "Integrated (I)" version of a stationary series.
} 


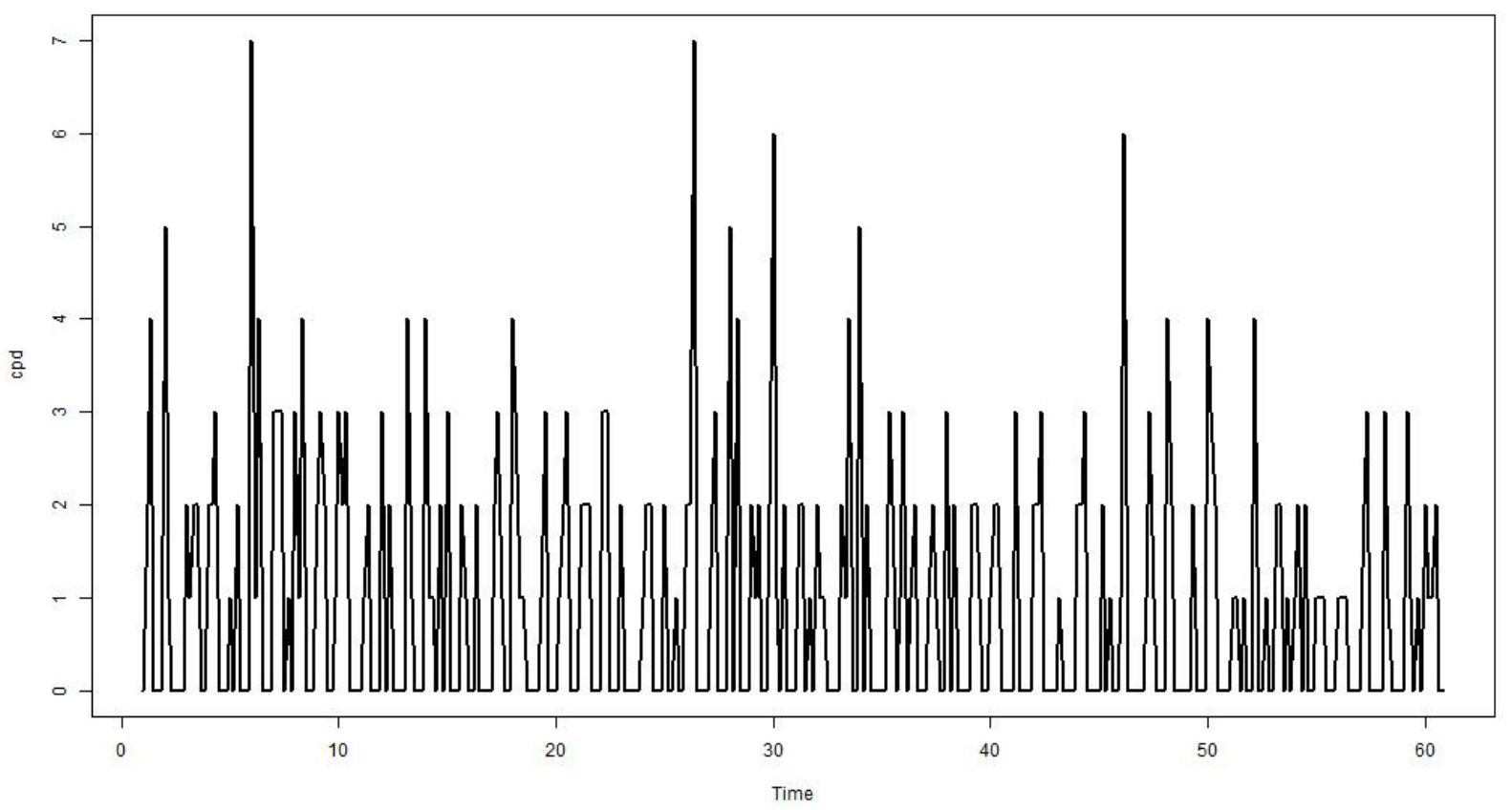

Fig. 9. Time Series of coffees prepared along 30 days within the laboratory.

\section{Model identification:}

Before a model is identified, a first step is to check if stationarity is present on the coffee's series to remove trend or scedasticity. ${ }^{17}$ To this aim Kwiatkowski et al. [29] developed a stationary test which takes into account the possible residual autocorrelations in the time series. This procedure tests the null hypothesis of level or trend stationarity against the unit root alternative. The result of the test is a $\mathrm{p}$-value of 0.022 which is lower than $\alpha=0.05$, enough level of significance to reject the null hypothesis, thus the series is not stationary and needs at least a difference. Figure 10 shows the $d=1$ differenced coffee's series and their associated ACF and PACF graphs. In a first approach to identify the appropriate model (identify the empirical auto-correlation patters - ACF and PACF - with the theoretical ones) we have observed that ACF is spiked at lags 1 and 2 and declines towards zero in lag 3 . It also shows that PACF is spiked at lag 1 while lag 2 is shrinking towards the confident bounds. With this information, we can speculate with a model similar to $\operatorname{ARIMA}(3,1,1)$.

Nevertheless, to select the best ARIMA fitting we have tested several models to the series. We have based our election in Akaike's Information Criterion (AIC) [30]. The AIC is used to compare competing models fit to the same series. The model with the smaller information criteria is said to fit the data better. The final selected model is $\operatorname{ARIMA}(3,1,1)(2,0,2)$. The latter set of this model refers to the ARIMA's seasonal part: $P$, the number of seasonal autoregressive (SAR) terms, $Q$ is the number of seasonal moving average (SMA) terms and $D$ is the number of seasonal differences. The seasonal part of an ARIMA model (also called SARIMA) has the same structure as the non-seasonal part. In this seasonal part, all the factors operate across multiples lags (the number of periods in a season). A SARIMA is

\footnotetext{
${ }^{17}$ Recall that as a first approach to eliminate cyclical fluctuations, we have separated the original series, whole work-day, into two parts: 6 hours for morning (7a.m.-13p.m.) and the next 6 hours for the afternoon.
} 

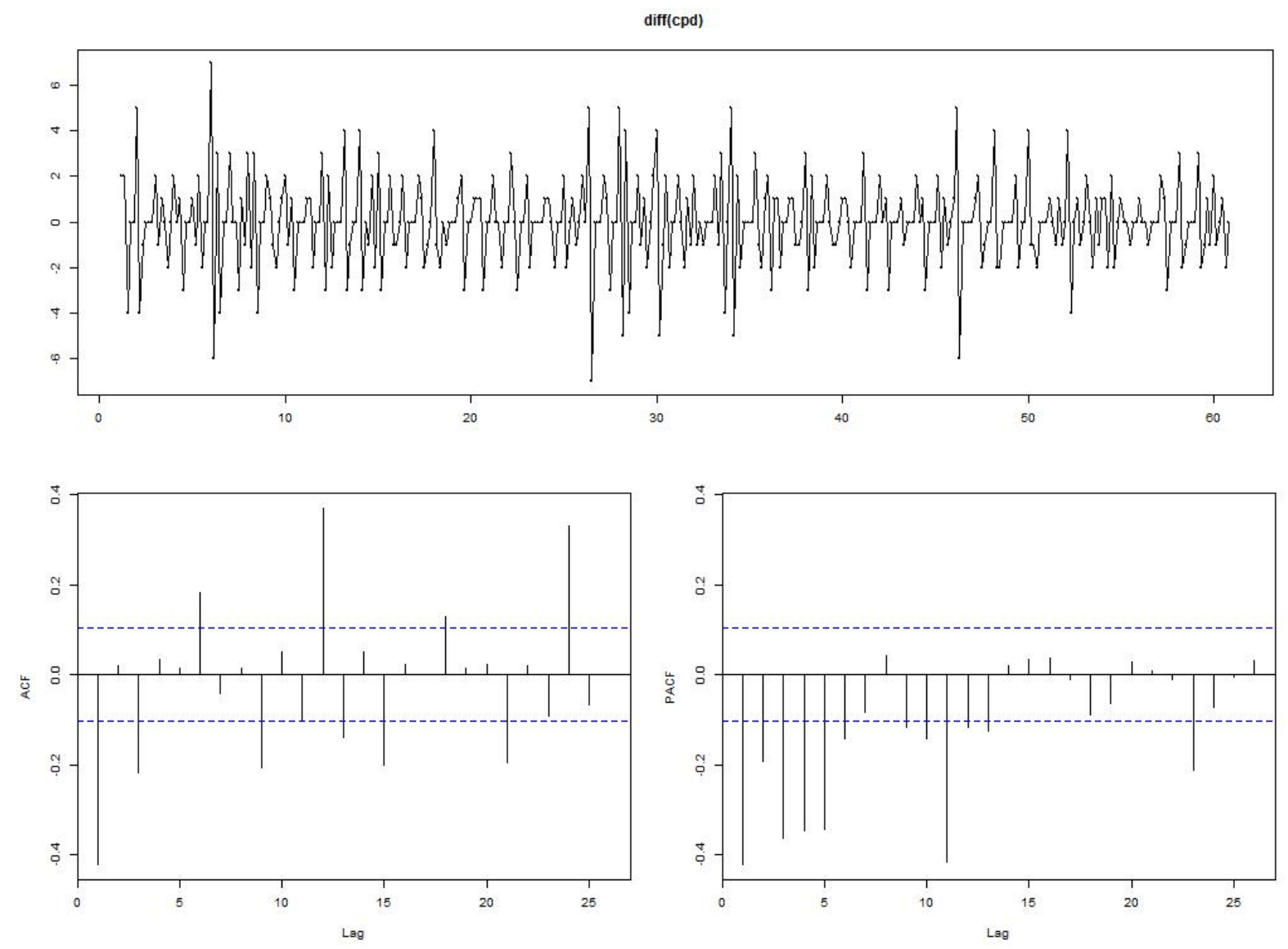

Fig. 10. Differenced series with its associated ACF and PACF for model identification.

therefore classified as $\operatorname{ARIMA}(p, d, q)\left(P_{1}, D_{1}, Q_{1}\right)$ and is formula is written as follows:

$$
\phi_{p} \Phi_{P_{1}}\left(L^{K_{1}}\right)(1-L)^{d}(1-L)^{D_{1}} y_{t}=\theta_{q}(L) \Theta_{Q_{1}}\left(L^{K_{1}}\right) \varepsilon_{t}
$$

In the Eq. (9), $K_{1}$ is the seasonal period; while $\Phi_{P_{1}}$ and $\Theta_{Q_{1}}$ are polynomial functions of order $P_{1}$ and $Q_{1}$.

Finally we plot the residuals on Fig. 11 and observe the goodness of the fit to check if the model assumptions are satisfied. The Figure shows that there is no significant autocorrelations left in the residuals from $\operatorname{ARIMA}(3,1,1)(2,0,2)$ model. This is known since these autocorrelations are concentrated within Bartlett's window edges. Besides, we obtain a p-value of 0.9391 (which is much bigger than the confident error $\alpha=0.05$ ) when performing the Box-Ljung test [31]. This fact also demonstrates that the model provides an adequate fit to the data, thus random residuals and they not present autocorrelation. Finally it is remarkable that the residual follows a normal distribution $\mathcal{N}(0, \sigma)$ with Gaussian noise.

\section{Model validation and forecasting:}

To validate the $\operatorname{ARIMA}(3,1,1)(2,0,2)$ we compare the forecast over the last 11 days of the coffeemachine's dataset with the real values observed during the forecast period. For the SARIMA model, we 
res
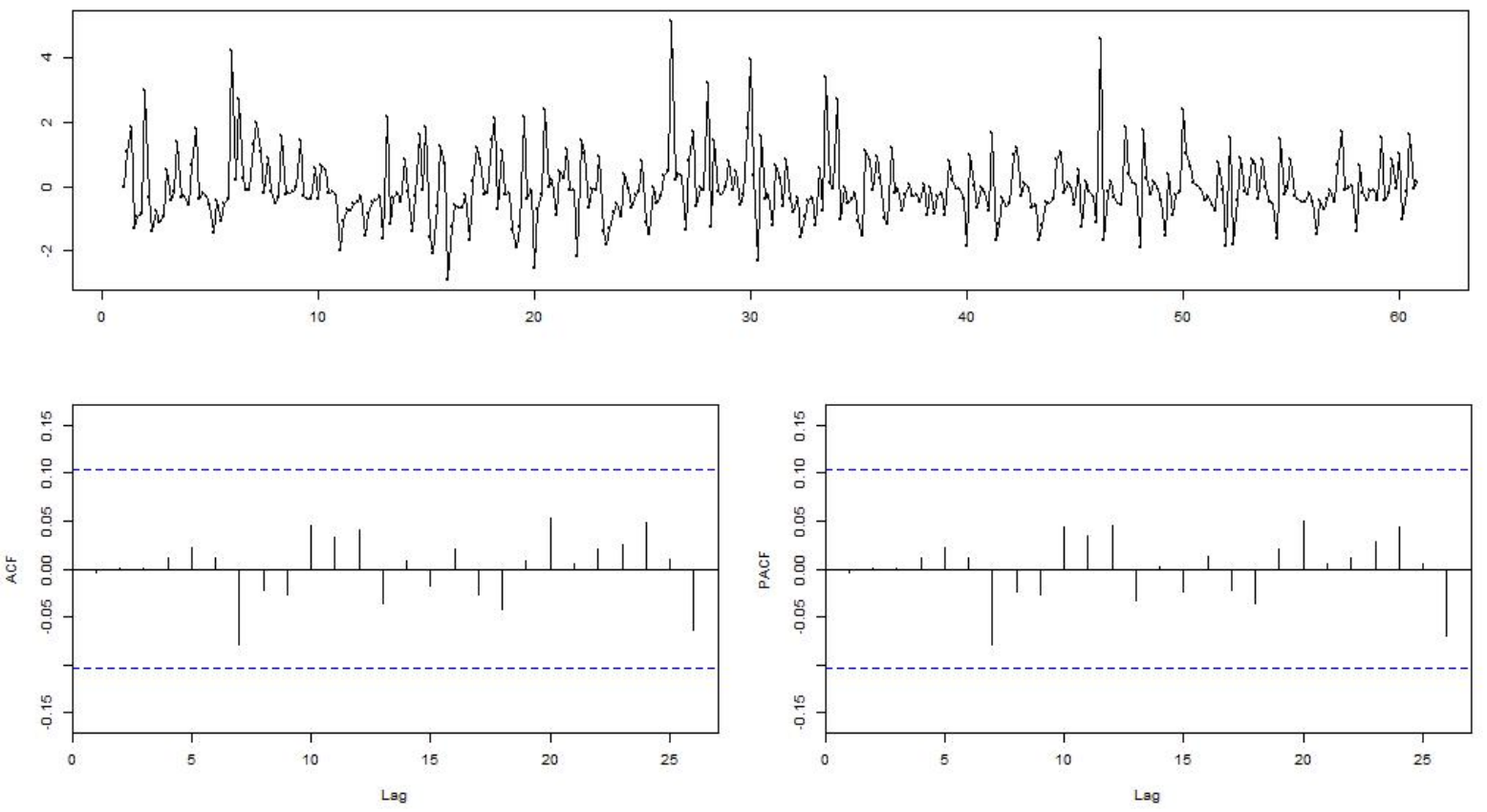

Fig. 11. Residuals and their associated autocorrelation graphs.

have averaged the prediction to the mean between the real forecast and the upper bound with $80 \%$ rate of confident.

As an example of this prediction, the Fig. 12 shows in blue the forecast values from the 31th to 41th days of the dataset. The green line correspond to the empirical data collected for the testing set (note that in this case the attained values for each of the evaluated periods ${ }^{18}$ are round). The remainder broken red-lines define the upper and lower confident bounds. The upper error bound has been calculated with 95\% confident and the lower bound of is stead to 0 coffees (according with the prediction, the expected number of coffees prepared will never exceed the upper bound and will not exhibit negative values). Without analyzing deeply the graphical plot, we can assert that the prediction is not far away to the real case. In the next Section we review quantitatively this results, in terms of energy savings, and we compare this promising approach with the related predictive models.

\section{Analysis and discussion}

To be able to analyze the quality of the different methods results, in terms of energy consumption, we have plot the Fig. 13. There, we have compared the proposed learning approaches with the real consumption (which has been used as baseline), with the Auto-power down mode consumption (which is the mode boosted by the EU for new manufactured appliances), and with the Optimal mode consumption (which is the ambitious goal that we are pursuing).

\footnotetext{
${ }^{18}$ As explained before everyday is divided in two parts: 6 hours in the mornings, from 7 a.m. to 1 p.m., while the same applies in the afternoon, i.e. from 1 p.m. to 7 p.m.
} 


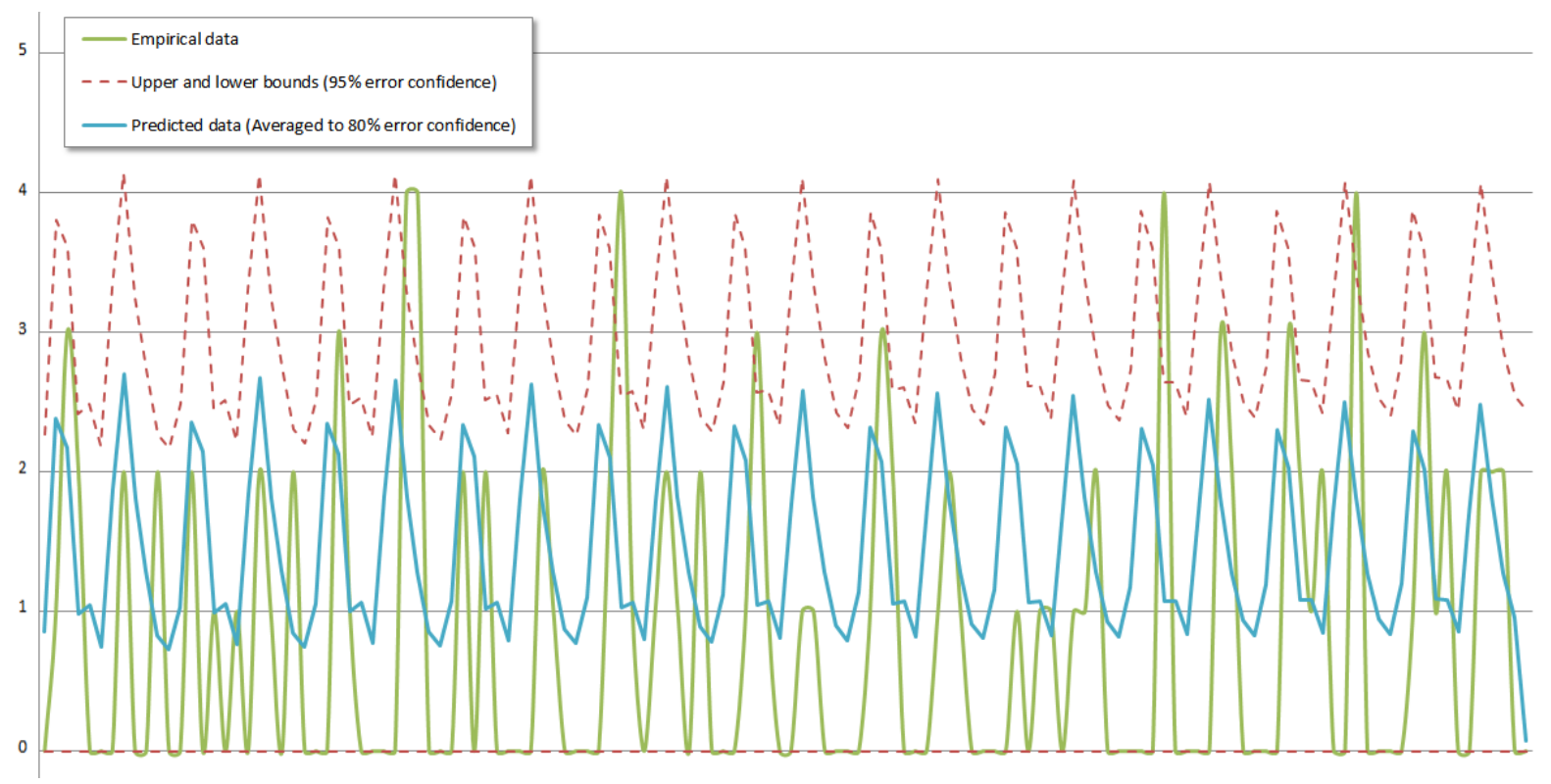

Fig. 12. Prediction of coffees prepared in next 11 days after model identification in day 30th.

The Auto-power down mode has been simulated establishing the rule, that when coffee machine detects a 30 minutes period of inactivity, it automatically switches off. We have also simulated the Optimal mode determining that the coffee-maker would be always operating in On-Off mode, except in slots where the number of coffees prepared exceeds 2.3, that it would shift to Stand-By mode.

As Fig. 13 shows, the proposed three predictive models save energy when compared with the baseline. The on-the-shelf Eco-mode which is programmed in the coffee-maker (Auto-power down mode) also saves more energy than the baseline. However, due to its general purpose design, it is not capable to dynamically elucidate an optimal operation depending on external features (like the number of people that will use the appliance). We can see that the presented machine learning methods achieve lower consumptions than it. The results of the proposed approaches are still far from the Optimal mode. This is due to a significant difference of the coffee maker usage pattern between the training set and test set (surprisingly in slots when was very common to prepare more than 3 coffees in the training phase, not more than 1 or 2 cup of coffees were filled in the test set. It occurs the same in the opposite case, i.e. more coffees were prepared in slots where the threshold was never exceed.).

Although we would have improved the inference of the coffees prepared by modifying the dataset order, we have kept rigorous with the historical sequential data that one of the proposed models imposes. While the dataset is not as propitious as we desire, we can see that the energy saving is still significant, saving $13.56 \%$ of the real energy consumption with heuristic approach, $14.02 \%$ with ARIMA model and $15.18 \%$ with Naïve Bayes model. Extrapolating this results to one year, the energy saving would rise to $8 \mathrm{kWh}$. According with General Electric web-site ${ }^{19}$ the same coffee machine could prepare more than 80 coffees with such amount of energy (i.e. roughly the same number of coffees prepared in our laboratory during two weeks).

\footnotetext{
${ }^{19} \mathrm{http}: / /$ visualization.geblogs.com/visualization/appliances/.
} 
Table 1

Comparison of the machine learning methods and real usage

\begin{tabular}{lccccc}
\hline Method & Total energy consumption & RMSE & MAE & MASE & Energy saving \\
\hline Heuristic approach & $2.24 \mathrm{kWh}$ & 0,4767 & 0,2272 & 1,3533 & $13.56 \%$ \\
Naïve Bayes & $2.20 \mathrm{kWh}$ & 0.3482 & 0,1212 & 0,7218 & $15.18 \%$ \\
Time Series (ARIMA) & $2.23 \mathrm{kWh}$ & 0,4924 & 0,2424 & 1,4435 & $14.02 \%$ \\
Real Usage & $2.60 \mathrm{kWh}$ & - & - & - & - \\
\hline
\end{tabular}

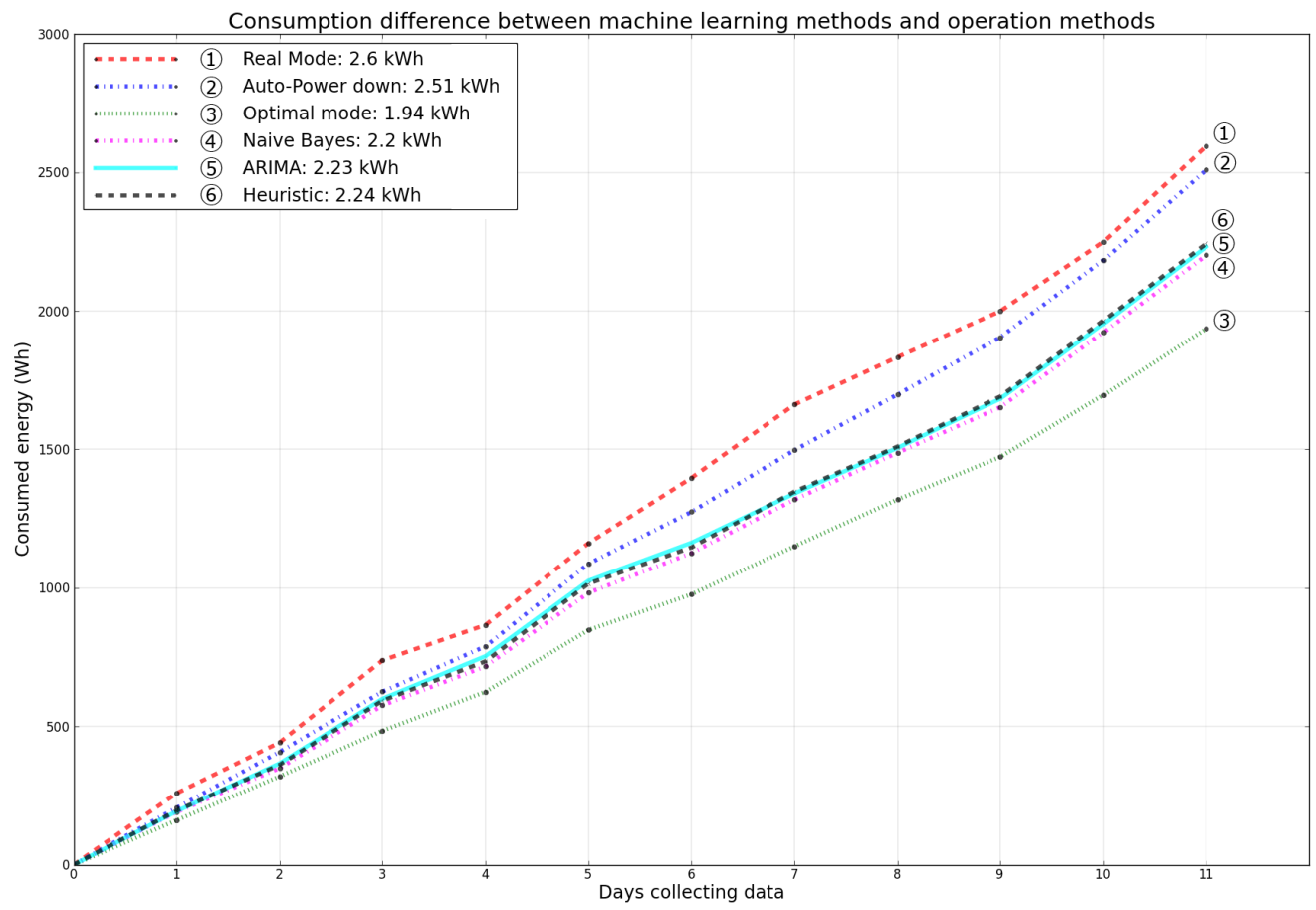

Fig. 13. Comparison of the accumulated energy consumption between methods.

Besides the comparison of energy saved by each of the predictive models, the Table 1 also evaluates their forecasting performance. To this aim, we have selected three accuracy measures such as MAE, RMSE and MASE that have been largely applied in similar prediction approaches. To calculate the errors uniformly, we have simplified each of the model predictions into a discrete election of modes (i.e. to leave the device on or off in a specific slot time). Then, we have compare such election with the real case. The selected error measures exhibit better performance if their values are low. Therefore, it can be said that the best model is clearly the Bayesian approach, while the Heuristic approach seems to be a little more successful in the forecasting when compared to ARIMA. Although the quantitative results show that ARIMA's performance is the worst, we argue that the errors calculated have been biased for the simplification of the model (please note that we have somehow transformed an approach that forecast real-values into a kind of binary predictive model).

The dataset that we have used only span a short period of time. Indeed, this data only disclose information about the coffee consumption and its associated energy in winter months. However, we think that the collection of usage-data in different periods along the year would entail different consumption patterns. This assumption have two major implications: 1) The selected predictive model must not remain 
static, so periodical recalculations and retraining are mandatory to accommodate to current usage. 2) It is pivotal to devise evaluation methodologies to prove if eco-behavior change is occurring (a diminution of energy waste could be due to conservation approaches rather than a decrease of coffee intakes).

A future step to achieve is to evaluate the appropriate periodicity in which the model should be recalculated. The first approach that we are going to test is every 24 hours (many literature strategies advocated for next-day prediction). However in the case of the coffee maker, this could yield to inconsistencies if the previous day exhibit unusual usage patterns.

In the article we have also explored the human behavior change strategy to reduce energy. This option has been largely approached in the literature through feedback techniques, but we contributed in a different way by giving a 'green-voice' to smart-everyday objects for promote sustainable behavior change through Social Networks. The results for this strategy are still immature, but we think that a combination of both: more Eco-aware people with more intelligent and sustainable devices, like our coffee-maker, is an enhanced approach. Furthermore, we can assert that the social coffee maker have attracted the interest of the majority of our laboratory-colleagues and even related people that not use the appliance at all (friends, researchers with similar interests, and also university students and professors). We expect that this popularity will be transformed in more discussions about energy concerns, i.e. increase e-awareness, and to rise the attention of interested users about the appropriate use that have to be applied to shared appliances, i.e. reduce misusing.

\section{Related work}

The energy savings that a predictive model for device operation, as a function of its real usage, can entails is a novel topic. Indeed, the majority of reviewed investigations deal only with the prediction of overall energy load for the next-day, but without taking into account neither the user preferences nor the real usage pattern of specific appliances in houses or offices. Nevertheless there are three approaches which are similar to the technical strategies that have been proposed in this article.

In [32], an automatic scheduling methodology for the efficient usage of shared devices in an industrial or commercial building is proposed. Their approach relies in the explicit user requests for collective appliance-use at different times, during a fixed interval (e.g. a work-day), based on dynamic energy prices during such interval. Although an electricity bill minimization problem is pursued, their approach assumes some sort of order in the tasks and device usage, while in our case this usage is completely stochastic and therefore inferred. Another related research is introduced by Barbato et al. [33]. Their approach which aims to devise strategies for dynamic energy pricing is fully-based on the continuous collection of data provided by sensors attached to appliances. The goal is to forecast every 24 hours which devices will be used on the next day, at what time, and for how long. They use the collected information for load demand management. Our approach goes beyond it since, besides forecasting the device's usage, we automatically use this information to switch between operating modes to save energy. Furthermore, while we propose machine learning methods (ARIMA and Bayessian), that are demonstrated to perform appropriately for forecasting, they only apply one probabilistic approach to predict the device's activation/operation (i.e. the sum of probabilities of the historical samples). The last article from Geurts et al. [34] describes an approach which applies machine learning techniques, taking into account also temporal attributes, for the inference of rules for switching control. Despite they focus in big system switching issue (voltage collapse detection and loss of synchrony in power interconnection), and they apply decision trees for inference, the machine learning problem is similar to ours. Thus, infer whether is appropriate or not to perform a switching operation for a specific purpose. 
Focusing in the methods that we have applied to predict when to intelligently switch between operating modes, we have realized that most of the reviewed articles use temporal machine learning techniques (specifically ARIMA models). However, these similar approaches tend to deal with macroeconomic predictions rather than small size homes' or offices' loads as our case study. The investigations which focus their predictions on the energy field, as is our case, aim to price forecasting [35], and alternatively, to energy load calculation [36], which is also a correlated measure to also predict energy prices. These studies discuss the appropriateness of applying ARIMA models versus other approaches such as Artificial Neural Networks (ANN) or regressions models, to the specific problematic that they face. A general conclusion is that ARIMA performs well in the prediction of linear time series but poorly in non-linear problems. To overcome this issue some studies propose the combination of ANN and ARIMA as a hybrid approach [36].

Meanwhile, Naïve Bayes has proven effective over the years in many applications, including text classification, medical diagnosis and systems performance management [37,38]. Closer to our problematic, this machine learning method has also used to predict the energy usage at home [39]. Taking into consideration, that Naïve Bayes is a probabilistic classifier, all the challenges have to be solved reducing them into a classification questions.

Finally in the field of behavior change, we have just found two articles which addressed the problematic of energy awareness in public spaces (e.g. at work). The most relevant to our research is [40]. Here the authors introduced several smart metering technologies in a large organization to observe their contribution in supporting sustainable energy practices at work. Although the paper is mainly an ethnography study of the role of workers in energy conservation, we have been highly motivated for it. They assert that with just a little awareness of energy leakages, the workers started to identify appliances with high standby energy consumption, and started to turn them off. Furthermore, one of their findings was that they noticed that awareness about energy consumption of collective goods was relative low compared to appliances in the area of personal responsibility. It is hard to compare our approach with them since they only give qualitative results. But we deem that Eco-aware and social devices should outperform the behavior change that Schwartz et al. experienced with dump energy meters. The other remarkable article is the Imprint [41]. It is an augmented printer (as well as our coffee-maker a shared device in public spaces), that displays in a close screen the data extracted from a printer queue (e.g. energy consumption or paper consumption). Identically as our approach they display the time that the printer is working versus its idle time. The authors aim is to open discussion and reflection among workers regarding the displayed data rather than modify the eco-behavior of their colleagues. Our investigations are very close, but the presented article goes beyond worker behavior change by making the Eco-aware devices not only mere reporters but eco-activist to reduce energy waste.

\section{Conclusions}

Experience has shown that humans use electrical appliances in public spaces less efficiently, in an energetic sense, than they should. Coffee machines are one of the most power hungry devices in the market. The stock of coffee machines in EU is 100 millions whereas a estimation of coffee machines in work-offices attains 30 millions. Thus means, whatever minimal energy reduction achieved with these devices will have a major impact in terms of energy and economy.

This paper has laid out the principles behind 'Eco-aware Smart Everyday Objects'. In our opinion, everyday collective objects should motivate people to have a more environmentally friendly behavior by making visible and understandable their energy waste. Although, earlier work has tackled this through 
feedback techniques, a more radical approach has been pursued by this work by giving a voice to smart everyday objects to participate in the energy conservation and thus promoting a sustainable behavior change through Social Networks. We have devised a proof of concept, in the form of a social intelligent coffee machine, to exemplify to future manufacturers of eco-appliances how they should improve their current static designs. The main idea behind is to make everyday objects even more efficient by enabling each of them operate differently as a function of their real usage pattern (i.e. automatically switching among available operating modes during the day, in order to save the most energy). In fact, this work has demonstrated that the proposed approach outperforms the traditional methods (e.g. Auto-power down mode) in terms of energy savings. A coffee machine is a good example of such commonly shared devices that should be more eco-friendly, since they expose high unnecessary energy consumption rates whilst idle.

The Internet of Things brings about inspiring opportunities where millions of connected objects come to participate in such ecosystem. Before this new technological advent, the percentage of energy used by ICT was astonishingly high compared with the overall consumption. A key goal of this work has been to alert to interested researchers, stakeholders and the general public about the ecological implications that this movement may cause (carbon footprint, silicon, energy for new connected devices, etc.). Most of previous research works have focused on energy-efficiency through low power transceivers or harvesting methods to cope with energy savings. This work takes a more ambitious approach, by making intelligent social devices to autonomously and intelligently prevent and react against energy waste taking into account their use by end-users.

The presented results validate our research hypothesis, i.e. "the active collaboration of people and Eco-aware everyday objects will enable a more sustainable/energy efficient use of the shared appliances within public spaces". Our experiments have shown that applying predictive models (Heuristics, ARIMA and Bayessian) allows to save respectively $13.56 \%, 14.02 \%$ and $15.18 \%$ of the total energy consumption when compared with the baseline $(2.60 \mathrm{kWh})$.

The future of this ongoing research is very promising. Next steps will be focused on evaluating other machine learning methods (e.g. ANN), improving the presented predictive models (e.g. performing a next-day prediction) and adequating the best solution issued on an embedded system within the coffee machine.

While regarding the behavioral strategies, we are going to collect more data to observe if behavior changes in the usage pattern according to different variables (e.g. season) occur. An interesting future step also is to achieve a bidirectional communication between humans and appliances to evaluate how it influences in energy terms.

A final interest is to elucidate whether the predictive mode would be extrapolated into other collective goods like printers, scanners or public screens with the same positive results.

\section{Acknowledgment}

The authors are very grateful to the University of Deusto for the financial support to their $\mathrm{PhD}$. studies.

\section{References}

[1] S. Foster Porter, L. Moorefield and P. May-Ostendorp, Energy Use of Plug Load Devices in California. Ecos Consulting Report. 31 Oct. 2006. 
[2] L. Blázquez, N. Boogen and M. Filippini, Residential Electricity Demand for Spain: New Empirical Evidence using Aggregated data. CEPE Working Paper No. 82. ETHZ. February 2012.

[3] J. Nipkow and E. Bush, Standby Consumption of Household Appliances. Swiss Federal Office of Energy, Zurich, Tech. Rep., Feb. 2003.

[4] A. Chakraborty and A. Pfaelzer, An Overview of Standby Power Management in Electrical and Electronic Power Devices and Appliances to Improve the Overall Energy Efficiency in Creating a Green World. In Journal Of Renewable and Sustainable Energy 3. 26th April, 2011.

[5] A.K. Meier and B. LeBot, One Watt Initiative: A global Effort to Reduce Leaking Electricity. Lawrence Berkeley National Laboratory Report \# LBNL 43954 [Online] Accessed on 25/10/2012 http://www.scientificcommons.org/6773465 (United States), 1999.

[6] B. Mohanty, Standby Power Losses in Household Electrical Appliances and Office Equipment. Regional Symposium on Energy Efficiency Standards and Labelling, Paris, France. 29-31 May, 2001.

[7] F. Mattern, T. Staake and M. Weiss, ICT for Green: How Computers can help us to Conserve Energy. In Proceedings of e-Energy 2010 pp. 1-10. Passau, Germany: ACM, Apr. 2010.

[8] G. Fitzpatrick and G. Smith, Technology-Enabled Feedback on Domestic Energy Consumption: Articulating a set of Design Concerns, In proceedings of IEEE Pervasive Computing 8(1) (Jan. 2009), 37-44.

[9] J. Morrish, Internet 3.0: the Internet of Things. Anaysis Manson Report. [Online] Accessed on 25/10/2012: http://www. analysysmason.com/Research/Content/Reports/RRY04_Internet_of_Things_Oct2010/ 11th October, 2010.

[10] G.E. Moore, Cramming more Components onto Integrated Circuits. Book Morgan Kaufmann, 2000, pp. 56-59.

[11] M. Weiser, The Computer for the Twenty-First Century, In Scientific American 265(3) (1991), 94-104.

[12] F. Siegemund, Cooperating Smart Everyday Objects - Exploiting Heterogeneity and Pervasiveness in Smart Environments, Ph.D. dissertation, ETH Zurich, Zurich, Switzerland, Dec. 2004.

[13] J. Bohn, V. Coroama, M. Langheinrich, F. Mattern and M. Rohs, Living in a world of smart everyday objects - social, economic, and ethical implications, In Journal of Human and Ecological Risk Assessment 10(5) (Oct. 2004), 763-785.

[14] J.I. Vázquez and D. López-De-Ipiña, Social devices: Autonomous Artifacts that Communicate on the Internet. In Proceedings of the 1st Int. conference on The IoT, ser. IOT'08 pp. 308-324. Berlin, 2008.

[15] Smart Metering Team, Towards a Smarter Future: Government Response to the Consultation on Electricity and Gas Smart Metering, Tech. Rep., Dept. of Energy and Climate Change. [Online] Accessed on 31/10/2012: http://decc.gov. uk/en/content/cms/consultations/closed/closed.aspx Dec, 2009.

[16] T. Graml, C. Loock and T. Staake, Online Energy Behavior Change Systems: What Makes People Stick? PERSUASIVE 2010? The Fifth International Conference on Persuasive Technology, Kopenhagen, Denmark, 2010.

[17] J. Froehlich, L. Findlater and J. Landay, The Design of Eco-feedback Technology. In Proceedings of the 28th international conference on Human Factors in Computing Systems (CHI'10), Atlanta, USA. April, 2010, pp. 1999-2008.

[18] J. Mankoff, J. Fussell et al., StepGreen.org: Increasing Energy Saving Behaviors via Social Networks. In Proceedings of the 4th International AAAI Conference on Weblogs and Social Media. May, 2010.

[19] M. Studley, S. Chambers, R. Rettie and K. Burchell, Gathering and Presenting Social Feedback to Change Domestic Electricity Consumption. In the 2nd International Workshop on Persuasion, Influence, Nudge and Coercion through mobile devices, May 2011.

[20] B. Josephy and J. Nipkow, Coffee Machines: Recommendations for Policy Design. In TIG (Topten International Group) [Online] www.topten.eu, Paris. May, 2012.

[21] E. Bush, J. Nipkow, B. Josephy, S. Heutling and R. Griesshammer, Strategies to Enhance Energy Efficiency of Coffee Machines. Topten International Group TIG, Swiss Agency for Efficient Energy Use S.A.F.E., German Federal Environment Agency, Oeko-Institute, EEDAL-Conference, Berlin 2009.

[22] J. Nipkow, Measuring Method and Calculation Formula for the Electricity Consumption of Coffee Machines for Household Use, Topten International Group TIG, Paris, France. May, 2009.

[23] J. López-de-Armentia, D. Casado-Mansilla and D. López-de-Ipiña, Fighting against Vampire Appliances through Ecoaware Things. In proceedings of The Sixth International Conference on Innovative Mobile and Internet Services in Ubiquitous Computing, 2012, pp. 868-873.

[24] I. Rish, An empirical study of the naive Bayes Classifier. In Proceedings of IJCAI-01 workshop on Empirical Methods in AI. Seattle, USA. 6th August, 2001.

[25] H. Zhang, The Optimality of Naive Bayes, In Proceedings of FLAIRS conference. Miami, USA. 17-19 May, 2004.

[26] R. Caruana and A. Niculescu-Mizil, An Empirical Comparison of Supervised Learning Algorithms. In Proceedings of the 23rd International conference on Machine Learning. Pittsburgh, USA. 25-29 June, 2006.

[27] N. Dikmen, Basic Concepts and Applications of Econometrics, Nobel Publication Distribution, Ankara 2009.

[28] G.E.P. Box and G. Jenkins, Time Series Analysis, Forecasting and Control, Holden-Day, Incorporated. 1990.

[29] P. Kwiatkowski, P. Phillips and P. Schmidt, Testing the Null Hypothesis of Stationarity Against the Alternative of a Unit Root: How Sure Are We That Economic Time Series Have a Unit Root? In Journal of Econometrics 54 (1992), 159-178. 
[30] H. Akaike, A new look at the statistical model identification, In IEEE Transactions on Automatic Control 19(6) (1974), 716-723.

[31] G.M. Ljung and G.E.P. Box, On a measure of lack of fit in time series models, In Biometrika 65 (1978), 297-303.

[32] S. Hatami and M. Pedram, Minimizing the Electricity Bill of Cooperative Users under a Quasi-Dynamic Pricing Model, In 1st IEEE International Conference on Smart Grid Communiations, Gaithersburg, USA, 2010, pp. 421-426.

[33] A. Barbato, A. Capone, M. Rodolfi and D. Tagliaferri, Forecasting the Usage of Household Appliances through power meter sensors for Demand Management in the Smart Grid, In Proceedings of IEEE International Conference on Smart Grid Communications (SmartGridComm), Brussels, Belgium, 2011, pp. 404-409.

[34] P. Geurts and L. Wehenkel, Temporal Machine Learning for Switching Control. In Proceedings of the 4th European Conference on Principles of Data Mining and Knowledge Discovery (PKDD ’00) Lyon, France, 2000, pp. 401-408.

[35] T. Jakasa and I. Androcec, Electricity Price Forecasting - ARIMA Model Approach. In proceedings of 8th International Conference on the European Energy Market (EEM), Zagreb, Croatia, 25-27 May, 2011, pp. 222-225.

[36] X. Wang and M. Meng, A Hybrid Neural Network and ARIMA Model for Energy Consumption Forcasting, Journal of Computers 7(5) (May 2012), 1184-1190.

[37] P. Domingos and M. Pazzani, On the optimality of the simple Bayesian classifier under zero-one loss, Machine Learning 29 (1997), 103130.

[38] J. Hellerstein, J. Thathachar and I. Rish, Recognizing end-user transactions in performance management, In Proceedings of AAAI-2000, Austin, Texas, 2000, pp. 596-602,

[39] C. Chen, B. Das and D. Cook, Energy prediction based on resident's activity. In Proceedings of the International workshop on Knowledge Discovery from Sensor Data, 2010.

[40] T. Schwartz, M. Betz, L. Ramirez and G. Stevens, Sustainable energy practices at work: understanding the role of workers in energy conservation. In Proceedings of the 6th Nordic Conference on Human-Computer Interaction: Extending Boundaries (NordiCHI '10). 2010, pp. 452-462.

[41] Z. Pousman, H. Rouzati and J. Stasko, Imprint, a community visualization of printer data: designing for open-ended engagement on sustainability, In Proceedings of the 2008 ACM conference on Computer supported cooperative work (CSCW '08), 2008, pp. 13-16.

Juan López-de-Armentia received his diploma in Computer Engineering in July 2008 from the Mondragon University. In 2009 joined Tecnalia Research Innovation and received his master degree in Control Engineering, Automation and Robotics in September 2011 from the University of Basque Country. In October 2011 he joined MORELab, a research group behind the Internet and Telecom units of DeustoTech - Deusto Institute of Technology at the Faculty of Engineering of the University of Deusto, where he is developing his PhD and works in topics related to Internet of Things, Energy Efficiency and Eco-aware Things.

Diego Casado-Mansilla is a PhD student at MORElab group within DeustoTech-University of Deusto in Bilbao, Spain. He received a MSc in Communication and Information Technologies from the University of Alcalá, Spain (2010) and a MSc in Research in Computer Science from the University of Rennes 1 (IFSIC), France (2009). His main research interests are focused in HCi Eco-feedback, persuasive technologies, smart everyday objects and sustainable behavior change.

Sergio Lopez Perez is a Junior Researcher/Adjunct Professor at DeustoTech (Energy Unit)/Department of Industrial Technologies, University of Deusto, Bilbao, Spain. He holds a degree in Mathematics from the University of Salamanca (2005), a MSc in Commercial Management and Marketing from EISE (2008) and a MSc in Development and Integration of Software Solutions from UDeusto (2011). His research interests are in optimization problems applied in the electricity and energy market, stochastic dynamic programming applied on transportation, forecasting, game theory, mechanism design theory.

Diego López-de-Ipiña is an associate professor and principal researcher of MORElab group (http://www.morelab.deusto.es/) associated to DeustoTech (http://www.deustotech.eu), University of Deusto in Bilbao, SPAIN. He is the director of the PhD program within the Faculty of Engineering. He received his PhD from the University of Cambridge, U.K in 2002. His main research interests are pervasive computing, internet of things, semantic service middleware, open linked data, social data mining and mobile-mediated and tangible human-environment interaction. He is currently taking part in several research projects applied to AmI-related application areas such as urban computing, sustainable computing or AAL. He has more than 100 publications in relevant international conferences and journals. 

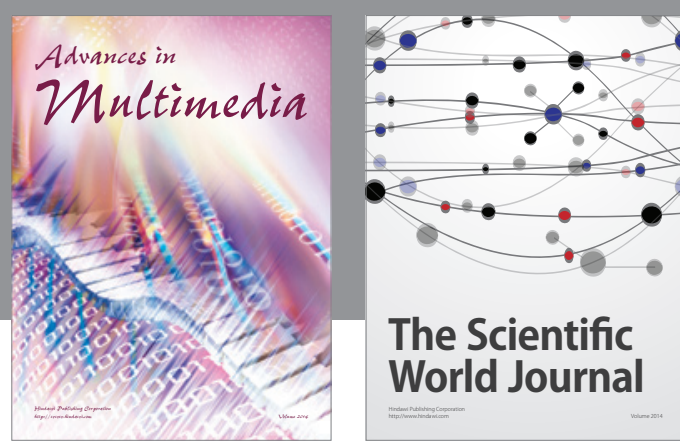

The Scientific World Journal
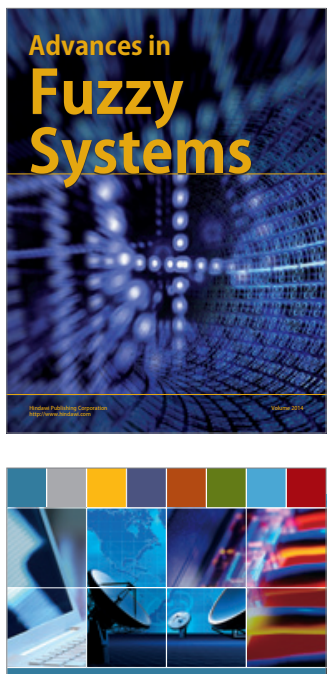

Computer Networks and Communications
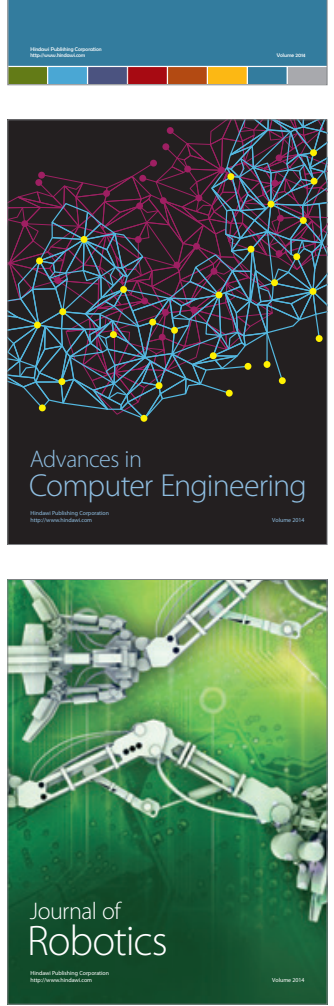
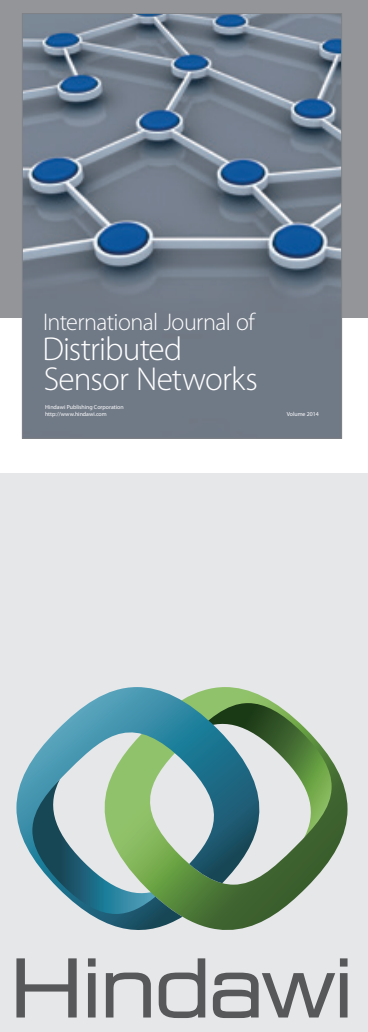

Submit your manuscripts at

http://www.hindawi.com
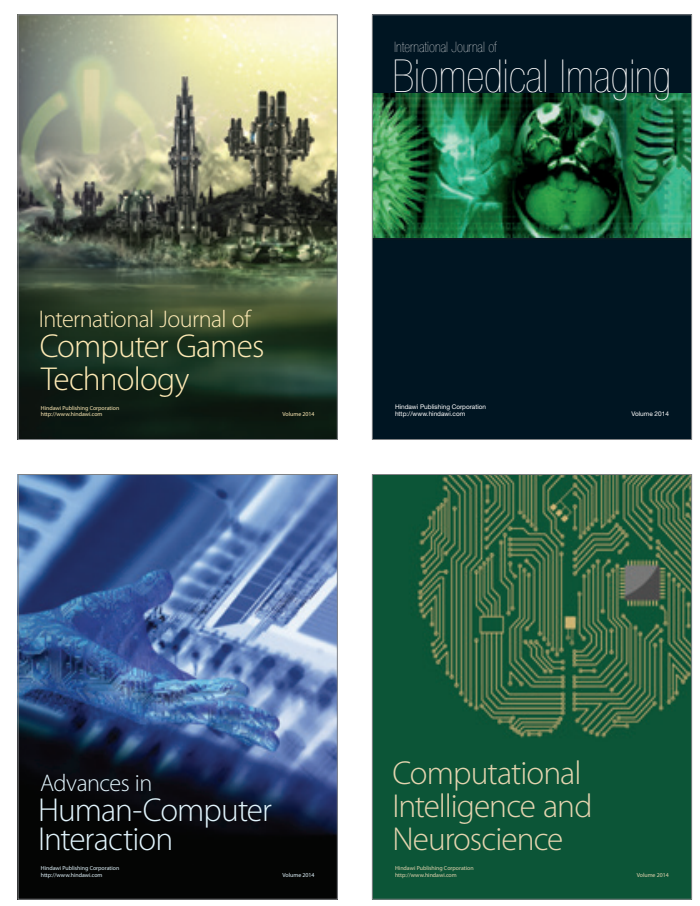
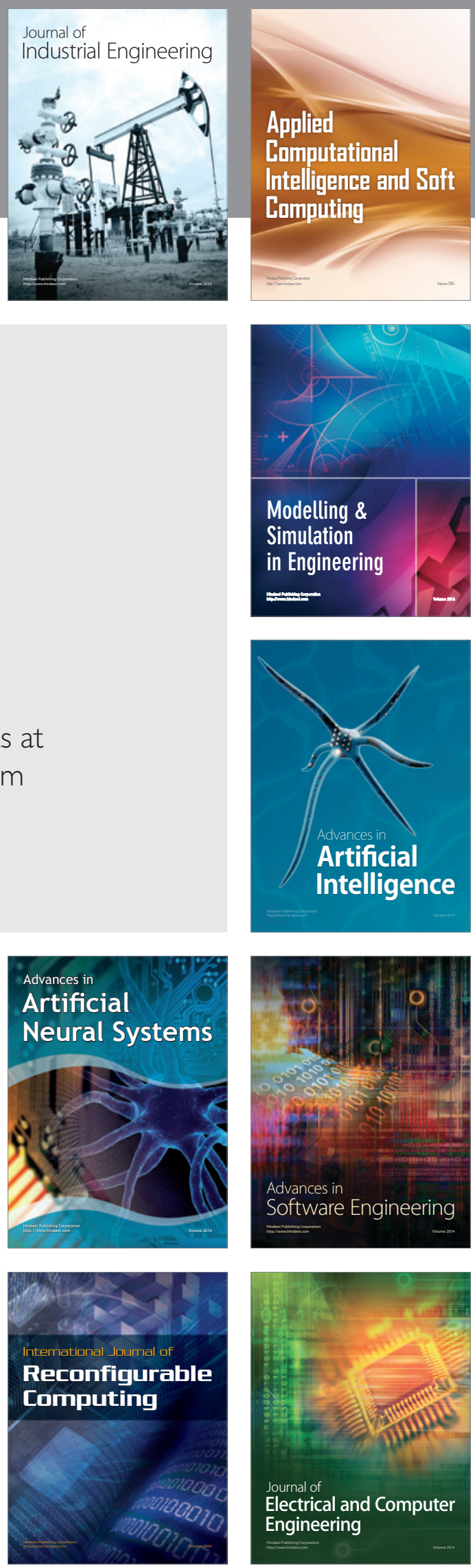